\title{
Smart Stimuli-Responsive and Mitochondria Targeting Delivery in Cancer Therapy
}

\author{
Yongjia Huang ${ }^{1}, *$ \\ Tingting Wang ${ }^{2, *}$ \\ Qunyou $\operatorname{Tan}^{3, *}$ \\ Dan $\mathrm{He}^{1, *}$ \\ Mingjun $\mathrm{Wu}^{4, *}$ \\ Jingchuan $\mathrm{Fan}^{4}$ \\ Jie Yang (D) \\ Cailing Zhong $\mathbb{D}^{\prime}$ \\ Kailing $\mathrm{Li}^{\prime}$ \\ Jingqing Zhang (D)' \\ 'Chongqing Research Center for \\ Pharmaceutical Engineering, Chongqing \\ Medical University, Chongqing, People's \\ Republic of China; ${ }^{2}$ Biochemistry and \\ Molecular Biology Laboratory, \\ Experimental Teaching and Management \\ Center, Chongqing Medical University, \\ Chongqing, People's Republic of China; \\ ${ }^{3}$ Department of Thoracic Surgery, Daping \\ Hospital of Army Medical University, \\ PLA, Chongqing, People's Republic of \\ China; ${ }^{4}$ Institute of Life Science, \\ Chongqing Medical University, \\ Chongqing, People's Republic of China \\ *These authors contributed equally to \\ this work
}

Correspondence: Jingqing Zhang Medicine Engineering Research Center, Chongqing Medical University, No. I, Yixueyuan Road, Yuzhong District, Chongqing, People's Republic of China Tel +8613308300303

Email zjqrae0I@I63.com

\begin{abstract}
Dysfunction in the mitochondria (Mc) contributes to tumor progression. It is a major challenge to deliver therapeutic agents specifically to the Mc for precise treatment. Smart drug delivery systems are based on stimuli-responsiveness and active targeting. Here, we give a whole list of documented pathways to achieve smart stimuli-responsive (St-) and Mc-targeted DDSs (St-Mc-DDSs) by combining St and Mc targeting strategies. We present the formulations, targeting characteristics of St-Mc-DDSs and clarify their anti-cancer mechanisms as well as improvement in efficacy and safety. St-Mc-DDSs usually not only have Mc-targeting groups, molecules (lipophilic cations, peptides, and aptamers) or materials but also sense the surrounding environment and correspondingly respond to internal biostimulators such as $\mathrm{pH}$, redox changes, enzyme and glucose, and/or externally applied triggers such as light, magnet, temperature and ultrasound. St-Mc-DDSs exquisitely control the action site, increase therapeutic efficacy and decrease side effects of the drug. We summarize the clinical research progress and propose suggestions for follow-up research. St-Mc-DDSs may be an innovative and sensitive precision medicine for cancer treatment.
\end{abstract}

Keywords: smart delivery, stimuli-responsive, mitochondria-targeting, tumor microenvironment, cancer therapy

\section{Introduction}

In addition to cancer prevention and early detection, an effective cancer treatment strategy is undoubtedly very important. ${ }^{1,2}$ In order to achieve maximum efficacy and minimal side effects, it seems to be necessary to precisely control drug location and maintenance at the destined tumor cells. ${ }^{3,4}$ Mitochondria (Mc) is linked to many hallmarks of cancer cells and its dysfunction may induce tumor cell death, so Mc has been considered as a pharmacological target over the last decades. ${ }^{5}$ Scientists have developed various smart stimuli-responsive (St) and Mc-targeting drug delivery systems (DDSs) (St-Mc-DDSs) (Figure 1). These systems have better efficacy and higher safety compared to Mc-DDSs or Sm-DDSs. ${ }^{5-10}$

St-Mc-DDSs have the added advantages of St-DDS and Mc-DDS (Table 1), including (1) hierarchical targetability. St-Mc-DDSs deliver drugs into tumor cells and cellular Mc organelles successively. (2) Controlled drug release. St-DDSs only release drugs at the target tumor tissue under the stimuli trigger but does not release or leak drugs at nontarget tissue sites. After entering the cells, St-Mc-DDSs further accurately deliver drugs to the target organelle Mc under the guidance of the Mctargeting group. (3) This system can achieve maximum therapeutic efficacy and produce minimal adverse effects. Drug molecules accumulate as much as possible 


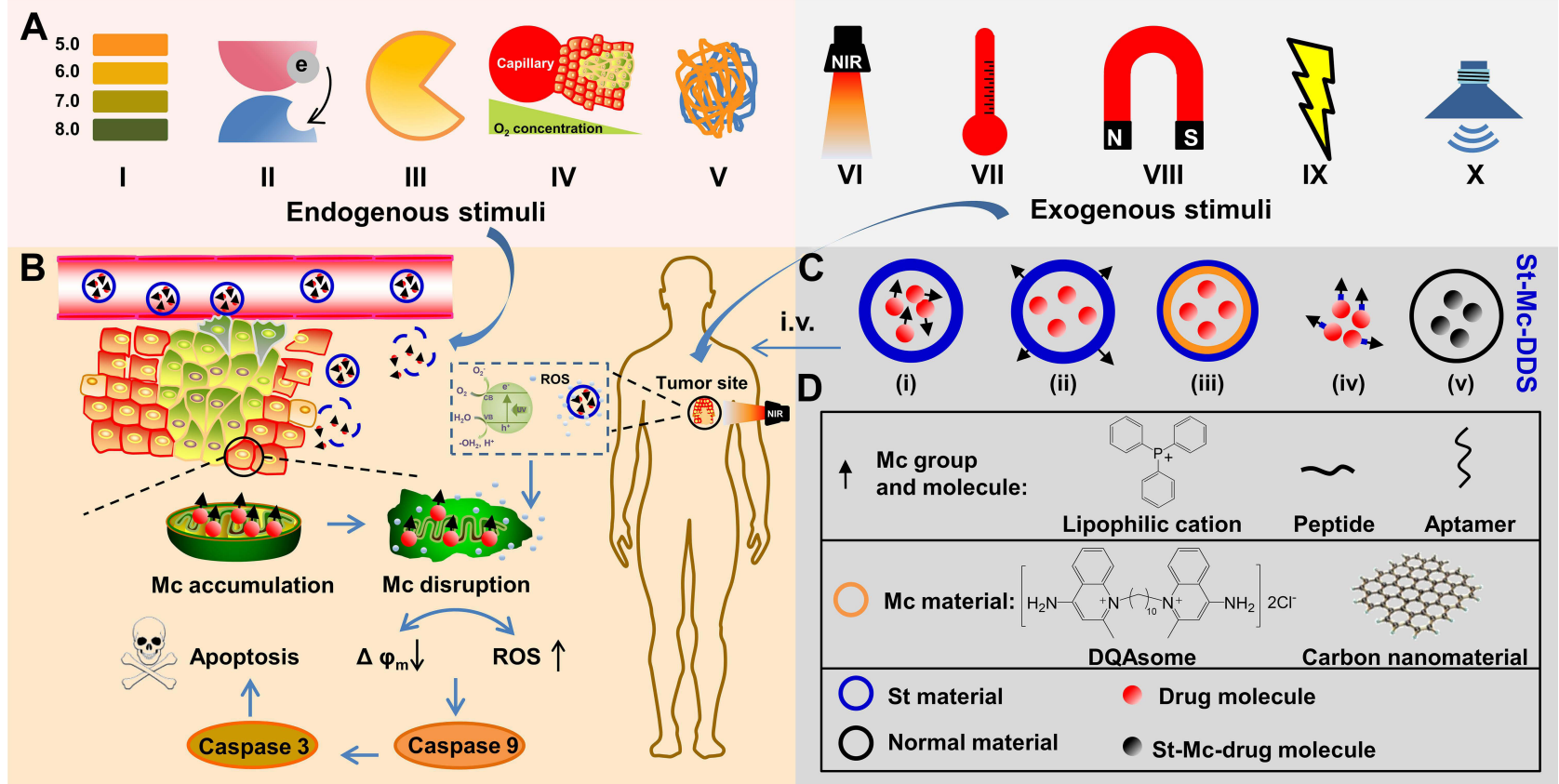

Figure I Schematic diagram of the therapeutic mechanism of St-Mc-DDSs.

Notes: (A) Endogenous and exogenous St types of St-Mc-DDSs. (B) Smart St-Mc-DDSs are achieved by combining St- and Mc-targeting strategies. (C) Five St-Mc-DDS types classified according to the spatial location relationships of different types of St- or Mc-DDSs. (D) Two types of Mc-targeting modes according to the structure.

Abbreviation: Mc, mitochondria; Mc-DDSs, mitochondria-targeting drug delivery systems; St, stimuli-responsive; St-DDSs, stimuli-responsive drug delivery systems; St-McDDSs, smart stimuli-responsive and mitochondria-targeting drug delivery systems.

at the precise target site organelle to exert efficacy while spending as little time as possible in normal cells and other organelles to reduce nonspecific toxicity. St-Mc-DDSs avoid the respective design based-deficiencies of the single St-DDSs (such as low drug concentration at the Mc) or Mc-DDS (such as high drug leakage at nontarget tissue/ cell).
The Mc presenting in most eukaryotic cells has been well recognized as an organelle target. ${ }^{11,12} \mathrm{The} \mathrm{Mc}$ is an organelle with two-layered membrane. It is approximately 0.5 to $1.0 \mu \mathrm{m}$ in diameter and of variable length. In addition to energizing cells, Mc also play a crucial role in cell differentiation, signaling and apoptosis. ${ }^{5,13}$ Mc dysfunction induces various hallmarks of cancer cells, such as

Table I A Comparison of the Characteristics Among St-Mc-DDS, St-DDS and Mc-DDS

\begin{tabular}{|c|c|c|c|}
\hline Comparison & St-Mc-DDS & St-DDS & Mc-DDS \\
\hline \multicolumn{4}{|l|}{ Advantage } \\
\hline Precisely Mc targeting & $\sqrt{ }$ & $x$ & $\sqrt{ }$ \\
\hline Maximum therapeutic response & $\sqrt{ }$ & $x$ & $x$ \\
\hline Stimulus responsiveness & $\sqrt{ }$ & $\sqrt{ }$ & $x$ \\
\hline Controlled drug release & $\sqrt{ }$ & $\sqrt{ }$ & $x$ \\
\hline EPR effect & $\sqrt{ }$ & $\sqrt{ }$ & $\sqrt{ }$ \\
\hline Long blood circulation & $\sqrt{ }$ & $\sqrt{ }$ & $\sqrt{ }$ \\
\hline Low clearance & $\sqrt{ }$ & $\sqrt{ }$ & $\sqrt{ }$ \\
\hline \multicolumn{4}{|l|}{ Disadvantage or deficiency } \\
\hline Low drug concentration at Mc & $x$ & $\sqrt{ }$ & $x$ \\
\hline High drug leakage at nontarget tissue/cell & $x$ & $x$ & $\sqrt{ }$ \\
\hline High non-specific toxicity & $x$ & $x$ & $\sqrt{ }$ \\
\hline
\end{tabular}

Notes: $\sqrt{ }$ Refers to have the property, $\times$ Refers to not have the property. 
limitless proliferation, damaged apoptosis, reduced autophagy and increased anabolism. ${ }^{14,15}$ However, Mc-targeted therapy is a tedious task.

Before the drug molecule finally reaches the $\mathrm{Mc}$, it needs not to be eliminated from blood circulation, selectively accumulate in the target tissue, pass through the cellular membrane barrier and escape from lysosomal endocytosis. ${ }^{5}$ The St-DDS seems to be able to increase the stability of the nanocarriers and reduce nontarget drug release in systemic circulation. The responsiveness of StDDS to endogenous (En) stimuli (also called biostimulators, include factors such as $\mathrm{pH}$, redox potentials, enzymes and glucose $)^{7,16,17}$ and exogenous (Ex) stimuli (also called externally applied triggers, include factors such as temperature, light and ultrasound) shows potential for a variety of biomedical applications ${ }^{8,18}$. The St-DDS senses the stimuli in the surrounding environment, analyzes the stimulus and responds accordingly. ${ }^{9}$ In reaction to the differences between tissue/cell microenvironments or blood systems of tumor and normality.

St-DDSs change the physicochemical characteristics (hydrophilicity, diameter or charge) accordingly to acquire greater in-depth tumorpenetration, increased cell ingestion, controlled drug delivery, and useful endosomal flee., ${ }^{7,9}$ Cellular Mc-specific DDSs may be achieved or strengthened by anchoring cell-specific homing ligands (which bind to overexpressed antigens on tumor cell outside) along with Mc-selective groups on its surface. ${ }^{19,20}$ The receptors currently utilized for active cancer cell targeted induction contain epidermal growth factor receptor (EGFR), ${ }^{21}$ folate $^{22,23}$ and transferrin receptors. ${ }^{24,25}$ For further endocytosis, a series of cell penetrating peptides (CPPs), such as cationic trans-activating transcriptional activator (TAT) ${ }^{26}$ polyarginine, ${ }^{27}$ amphipathic MPG (unabbreviated notation), ${ }^{28}$ Pep- $1,{ }^{29}$ Pep- $7,{ }^{30}$ and hydrophobic $\mathrm{C} 105 \mathrm{Y}^{31}$ have been developed to modify drug molecules or nanocarriers. After entering the organellerich cytoplasm, bioactive drug molecules need to be delivered directly to their Mc action site to acquire high therapeutic efficacy and low off-target result. ${ }^{10,32,33}$ The Mctargeted groups that are able to carry drugs include micromolecule ligands, ${ }^{34}$ hydrophobic cations, ${ }^{35,36} \mathrm{Mc}$ protein import machinery, ${ }^{37,38}$ Mc-penetrating peptides ${ }^{10,39}$ and Mc inner/outer membrane-targeting molecules. ${ }^{40,41}$ Through the above analyses, St-Mc-DDS deliver the medicines as much as possible to targeted organelle/cell/tissues and acquire perfect treatment effect and medication safety.
Obviously, there are many ways to combine St- and Mc- DDSs, and we have given a whole list of the documented pathways to achieve smart St-Mc-DDSs by combining St- and Mc-targeting strategies (Figure 2). Here, we systematically collected and analyzed the design theories, implementation method and action mechanisms of St-McDDSs in cancer therapy. We present their smart features and physicochemical and pharmacokinetic properties (Tables 2 and 3), which are closely related to their antitumor efficacy and safety (Tables 4 and 5). Compared to conventional DDSs, St-Mc-DDSs exhibit improved curative efficacy and safety. We propose the challenges and future perspectives of St-Mc-DDSs. This is the first review of the combined application of stimuli-responsive St- and Mc-targeted DDSs. This review will help to evaluate and choose the appropriate smart DDS to cure cancer.

\section{Mc Targeting Characteristics of St- Mc-DDSs}

St-Mc-DDSs usually have 2 types of Mc-targeting modes according to the component structure: an Mc group or an Mc molecule (lipophilic cation, peptide or aptamer), and a material (Figure 1D).

\section{Mc Group and Molecule-Based St-Mc- DDSs}

\section{Lipophilic Cation-Based St-Mc-DDSs}

Mitochondriotropics are low-molecular weight compounds with high intrinsic affinity towards Mc. Mitochondriotropic molecules often have delocalized positive charge and enough hydrophilicity. Once entering inside mammalian cells, mitochondriotropics accumulate either at or inside Mc without requiring the assistance of any Mc-targeted delivery system. ${ }^{82}$ Owing to the high Mc targeting of mitochondriotropics, they are widely used as Mc targeting groups to facilitate drug delivery. ${ }^{19,20,42,54}$ Hydrophobic cations such as triphenylphosphonium (TPP), ${ }^{42}$ (4-carboxybutyl) triphenylphosphonium bromide (CTPP), ${ }^{20}$ IR780 ${ }^{54}$ and 9-O-octadecyl ${ }^{19}$ have been used as Mc targeting groups of St-Mc-DDS to deliver a variety of small molecule drugs. TPP consists three phenyl groups. It has positive charge and sufficient lipophilicity that facilitates transportation across the Mc membrane. ${ }^{83,84}$ TPP and CTPP moieties are usually conjugated with small molecules $^{20,42}$ or decorated on the surface of nanoparticles. $^{43,44}$ Owing to their sufficient lipophilicity and delocalized positive charge, TPP-carrying molecules 


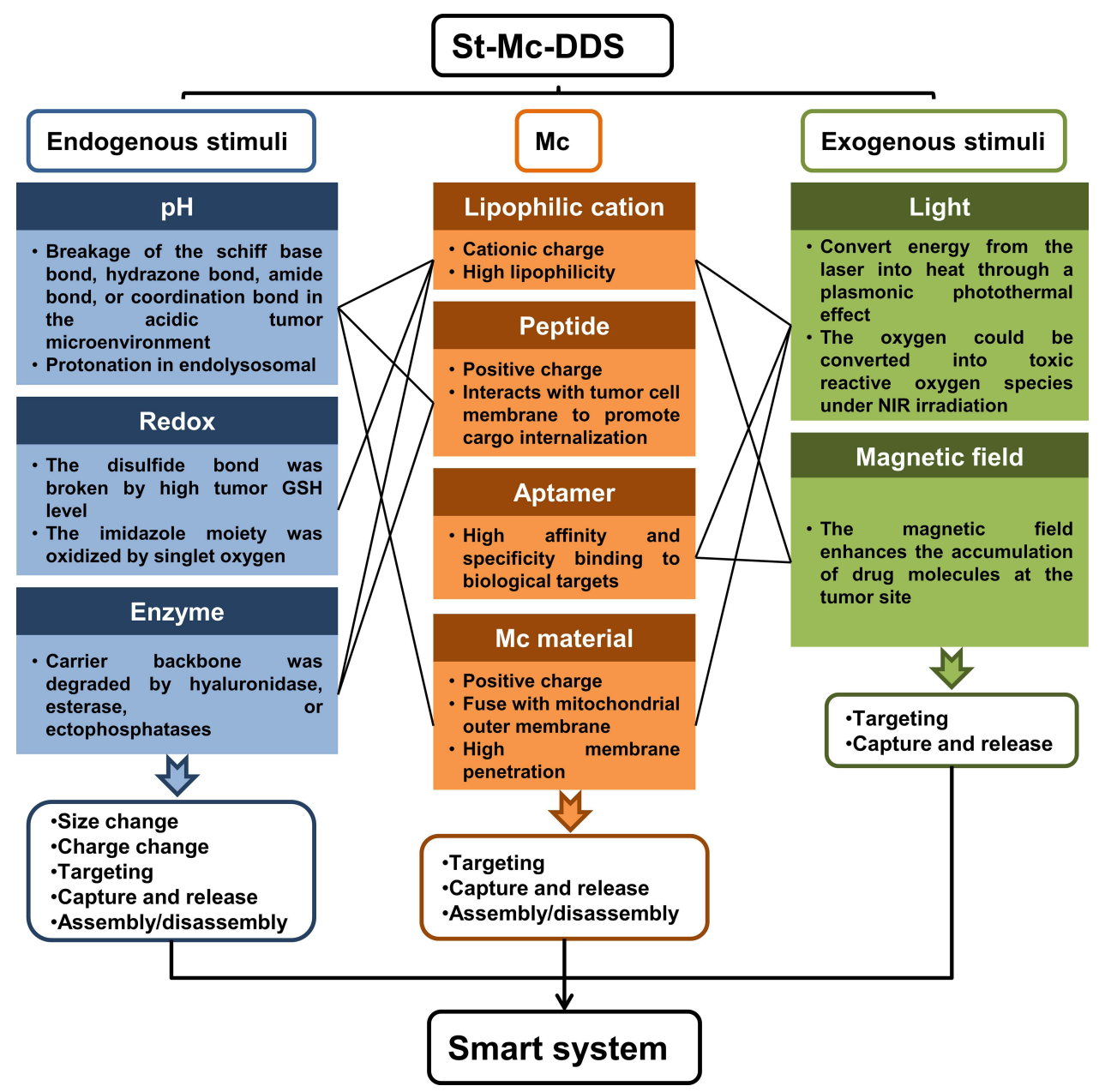

Figure 2 A list of documented pathways to achieve smart St-Mc-DDS by combining St- with Mc-targeting methods.

can get easily across lipophilic Mc membrane. ${ }^{85}$ The merits of TPP-molecules over other hydrophilic cation-molecules include its good biological stability, amphiphilic properties, ease of modification, inertia toward cellular materials, and deficiency in ultraviolet (UV)-visible light and fluorescence absorption. TPP-ubiquinone is safe for human, thus shows good clinical potential and the translational significance of TPP. ${ }^{34}$ IR-780 is a lipophilic heptamethine cyanine dye. It is automatically ingested by tumor cells through organic-anion polypeptide transporters, and accumulated in Mc sites due to its hydrophobic cation. ${ }^{86}$ IR-780 is typically used in nanocarriers for photothermography, photodynamic and photothermal therapies (PDT and PTT) ${ }^{54,56,86}$

In addition to the above lipophilic cations, rhodamine $123,{ }^{87,88}$ flupirtine, ${ }^{89}$ MKT- $077^{90}$ and anthracyclines $^{91,92}$ have demonstrated specific affinity to $\mathrm{Mc}$ in different Mc-DDSs, but they have not been used in St-Mc-DDSs.

\section{Mc Targeting Peptide-Based St-Mc-DDS}

Two Mc-targeting peptides, ie, KLA peptide (D $\left[\mathrm{KLAKLAK}_{2}\right)^{47}$ and MQ peptide (MLFNLRILLNN AAFRNGHNFMVRNFRCGQPLQ), ${ }^{37}$ have been used in St-Mc-DDSs for subcellular-targeted delivery of chemical drugs and DNA, respectively. KLA is an Mc-penetrating peptide (MPP) that can be decorated on the surface of liposomes. Positively charged KLA targets Mc, and its lysine unit facilitates cellular uptake since lysine interacts with the cellular membrane via a hydrogen bond and electrostatic force to accelerate internalization. ${ }^{47}$ In addition, KLA further disrupts the Mc membrane and initiates apoptotic cell death. ${ }^{93}$ MPPs have been confirmed to delocalize lipophilic cations to deliver bioactive compounds to the Mc. ${ }^{94-97}$ MPPs usually have positively charged (lysine, arginine) and lipophilic (isoleucine, phenylalanine, tyrosine) amino acids. ${ }^{98,99}$ For example, the aromatic cation-containing Szeto-Schiller (SS) peptide ${ }^{100}$ was mainly designed to deliver tyrosine or dimethyl tyrosine as an antioxidant motif 


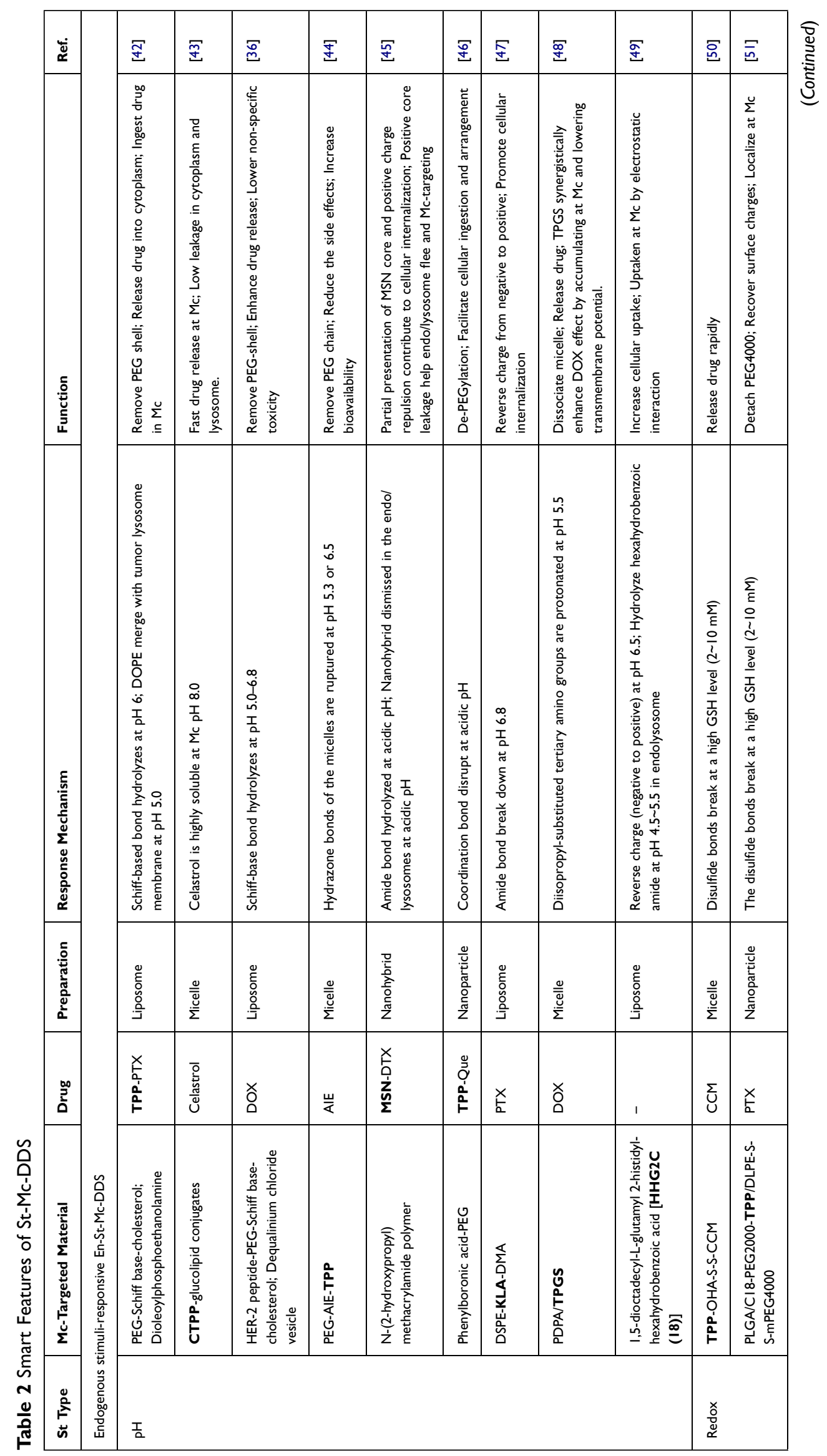




\begin{tabular}{|c|c|c|c|c|c|c|c|c|c|c|c|c|c|c|c|c|c|}
\hline$\dot{\mathscr{\varpi}}$ & 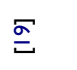 & స్ర & $\overline{\tilde{n}}$ & 㧀 & & 跑 & 氙 & 总 & $\sqrt{6}$ & 总 & ब్ & 잉 & $\underset{9}{F}$ & 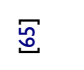 & 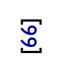 & है & ळ্口্ \\
\hline 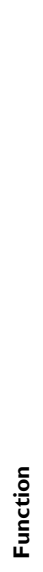 & 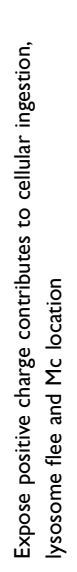 & 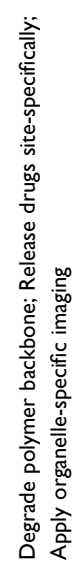 & 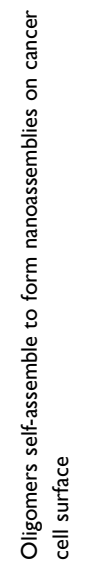 & 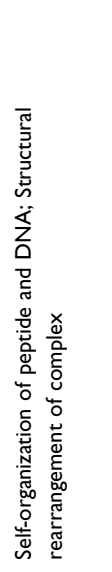 & & 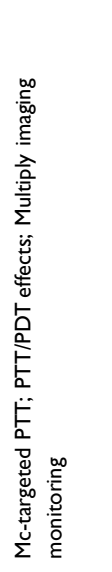 & 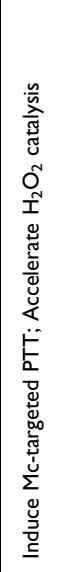 & 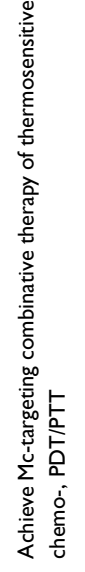 & 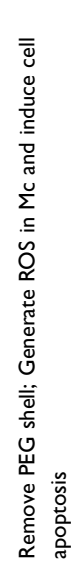 & 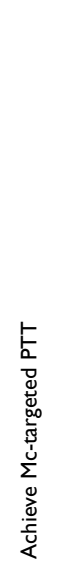 & 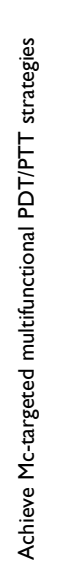 & 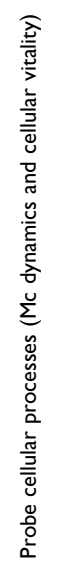 & 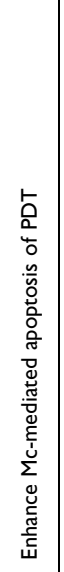 & 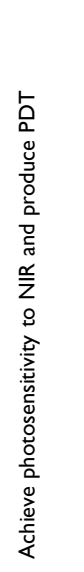 & 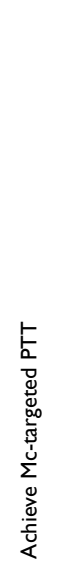 & 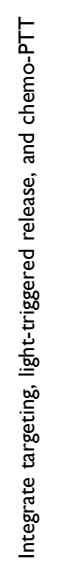 & 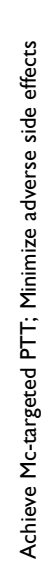 \\
\hline 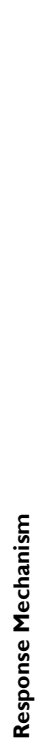 & 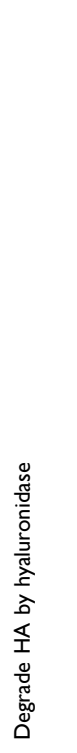 & 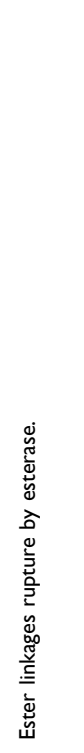 & 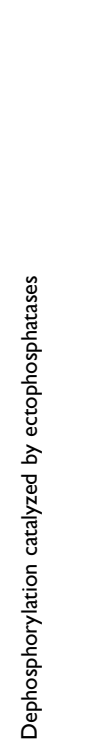 & 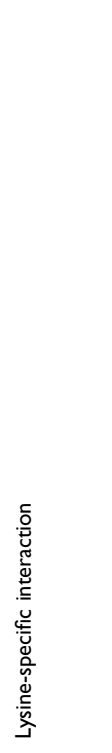 & & 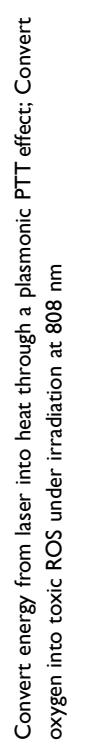 & 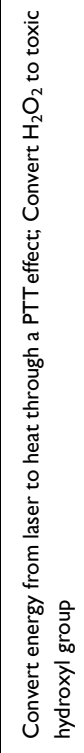 & 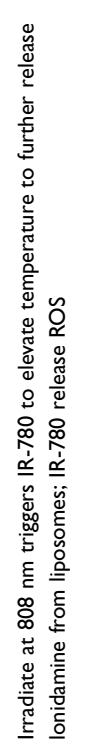 & 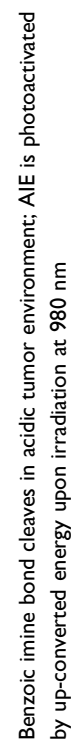 & 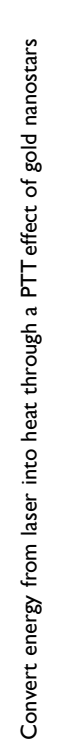 & 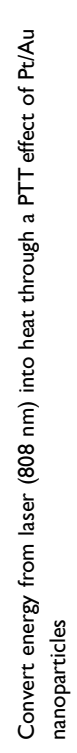 & 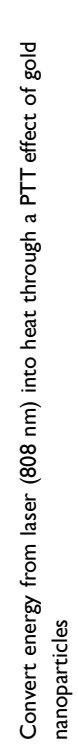 & 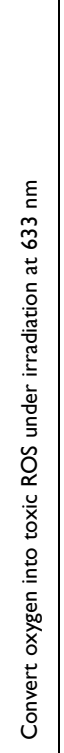 & 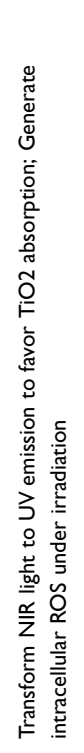 & 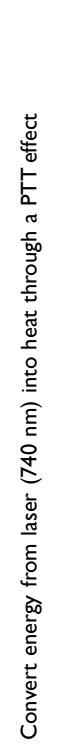 & 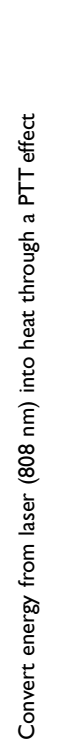 & 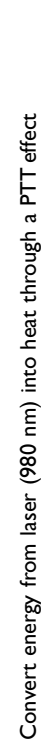 \\
\hline 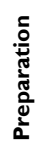 & $\begin{array}{l}\frac{\infty}{20} \\
\frac{2}{0} \\
\frac{0}{\tilde{I}} \\
\frac{\mathrm{T}}{Z}\end{array}$ & 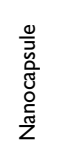 & 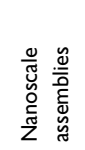 & 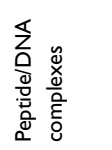 & & 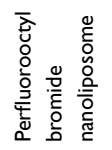 & 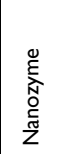 & 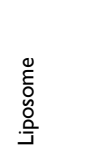 & 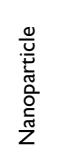 & 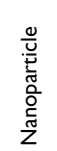 & 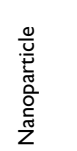 & 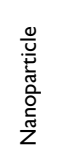 & 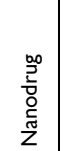 & 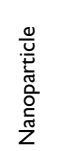 & 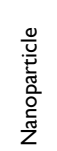 & $\begin{array}{l}\text { o } \\
\overline{0} \\
\frac{\bar{E}}{\tilde{T}}\end{array}$ & 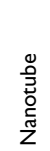 \\
\hline$\stackrel{2}{0}^{\infty}$ & 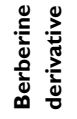 & ○ે & 贾 & $\begin{array}{l}\text { Zz } \\
\text { a }\end{array}$ & & $\begin{array}{l}\stackrel{D}{D} \\
\underline{\underline{a}}\end{array}$ & $\mid \begin{array}{l}\underline{E} \\
\underline{\underline{\underline{z}}} \\
\underline{\underline{\underline{z}}}\end{array}$ & 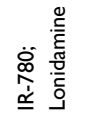 & $\frac{山}{\varangle}$ & 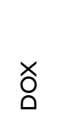 & $\begin{array}{l}z \\
\dot{x} \\
\vdots \\
0\end{array}$ & そ & $\frac{\pi}{0}$ & 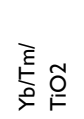 & $\begin{array}{l}O_{\tilde{\Phi}}^{+} \\
\tilde{W}^{+}\end{array}$ & そ & 攴 \\
\hline 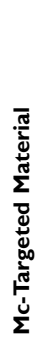 & 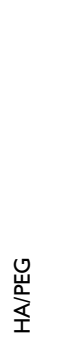 & 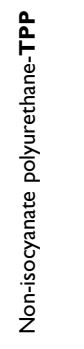 & 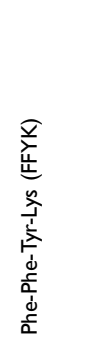 & 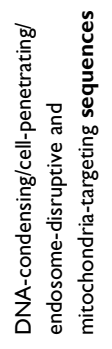 & 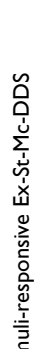 & $\begin{array}{l}\stackrel{D}{\Phi} \\
\underline{\underline{\underline{m}}}\end{array}$ & $\begin{array}{l}0_{0}^{+} \\
\mathscr{J}^{\circ}\end{array}$ & 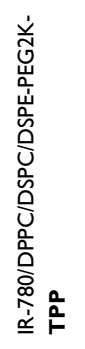 & 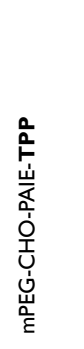 & 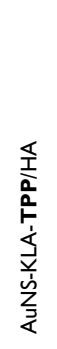 & 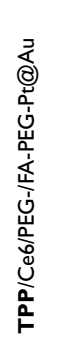 & 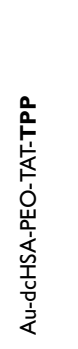 & 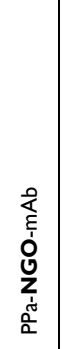 & 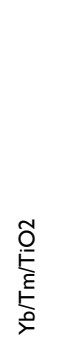 & 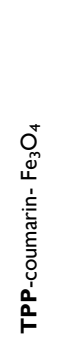 & 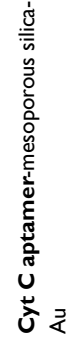 & 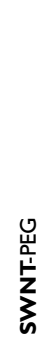 \\
\hline 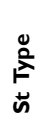 & \multicolumn{5}{|c|}{ 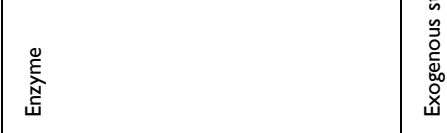 } & \\
\hline
\end{tabular}




\begin{tabular}{|c|c|c|c|c|c|c|c|c|c|c|}
\hline 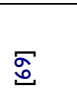 & $\bar{\Omega}$ & $\Sigma$ & & $\overline{\mathrm{d}}$ & $\Xi$ & $\overline{\underline{\varepsilon}}$ & $\underset{\Sigma}{\Xi}$ & I & 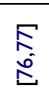 & $\stackrel{\infty}{\mathscr{\infty}}$ \\
\hline 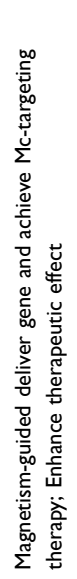 & 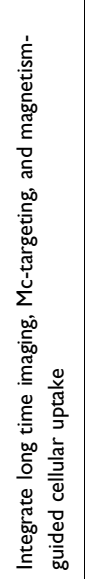 & 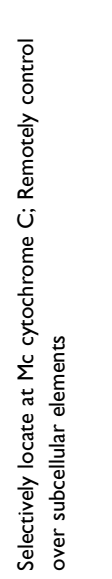 & & 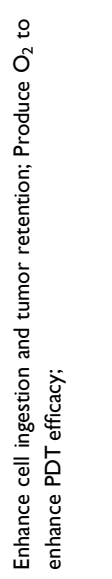 & 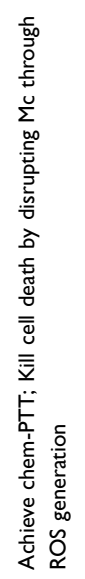 & 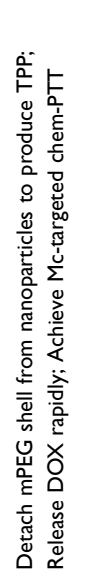 & 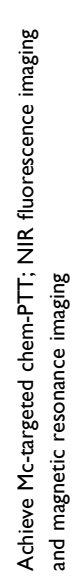 & 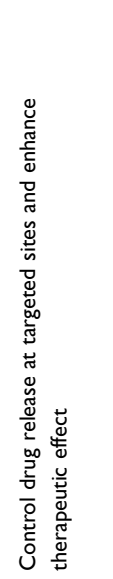 & 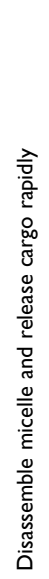 & 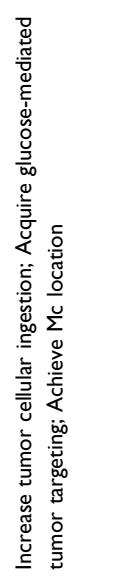 \\
\hline 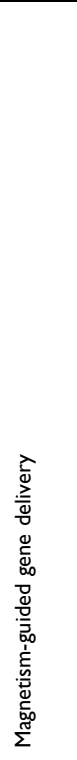 & 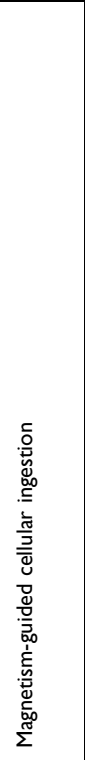 & 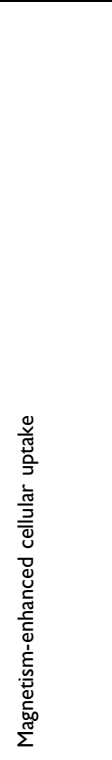 & & 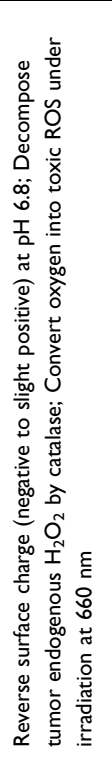 & 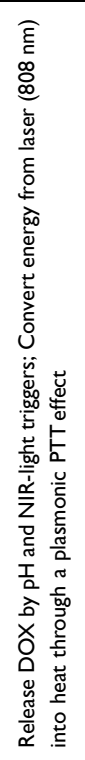 & 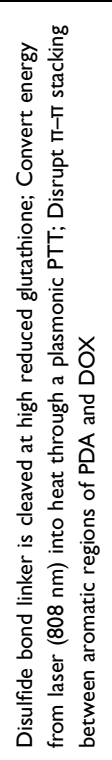 & 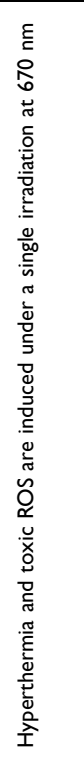 & 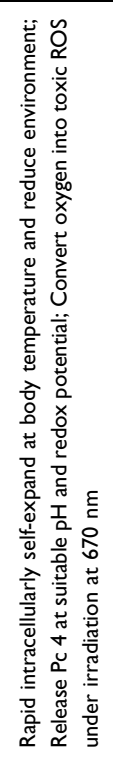 & 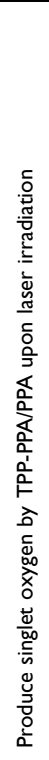 & 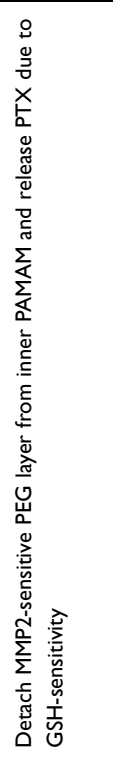 \\
\hline$\frac{z}{0}$ & 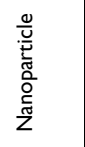 & 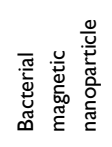 & \multirow{4}{*}{ 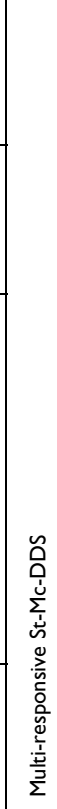 } & 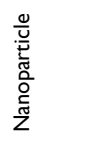 & 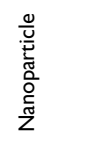 & 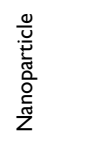 & 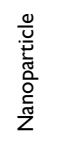 & $\begin{array}{l}\overline{\mathrm{d}} \\
\text { o. } \\
\overline{\mathrm{E}} \\
\overline{\mathrm{E}}\end{array}$ & $\begin{array}{l}\frac{\mathscr{\varrho}}{\overline{\underline{\Xi}}} \\
\frac{\bar{L}}{\Sigma}\end{array}$ & 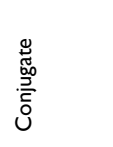 \\
\hline$\underline{z}$ & $\begin{array}{l}0^{+} \\
\tilde{D}^{\circ}\end{array}$ & $\overline{\dot{\omega}}$ & & 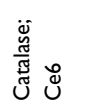 & 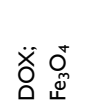 & $\begin{array}{l}\ddot{㐅} 0 \\
\text { Oे } \\
\text { ơ }\end{array}$ & 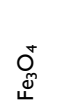 & $\stackrel{\square}{a}$ & 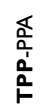 & 希 \\
\hline 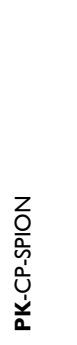 & 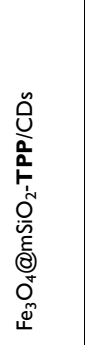 & 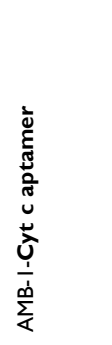 & & 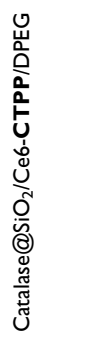 & 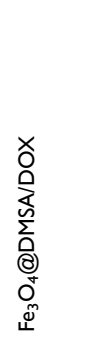 & 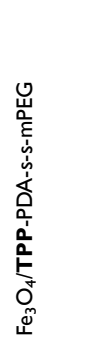 & 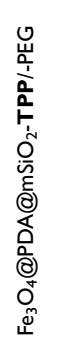 & 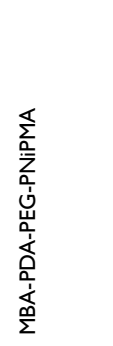 & da & 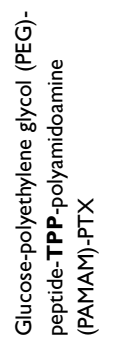 \\
\hline 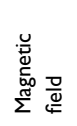 & & & & \multicolumn{2}{|l|}{ 囍 } & 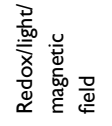 & 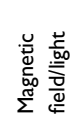 & 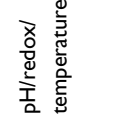 & 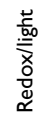 & 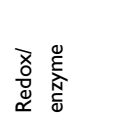 \\
\hline
\end{tabular}




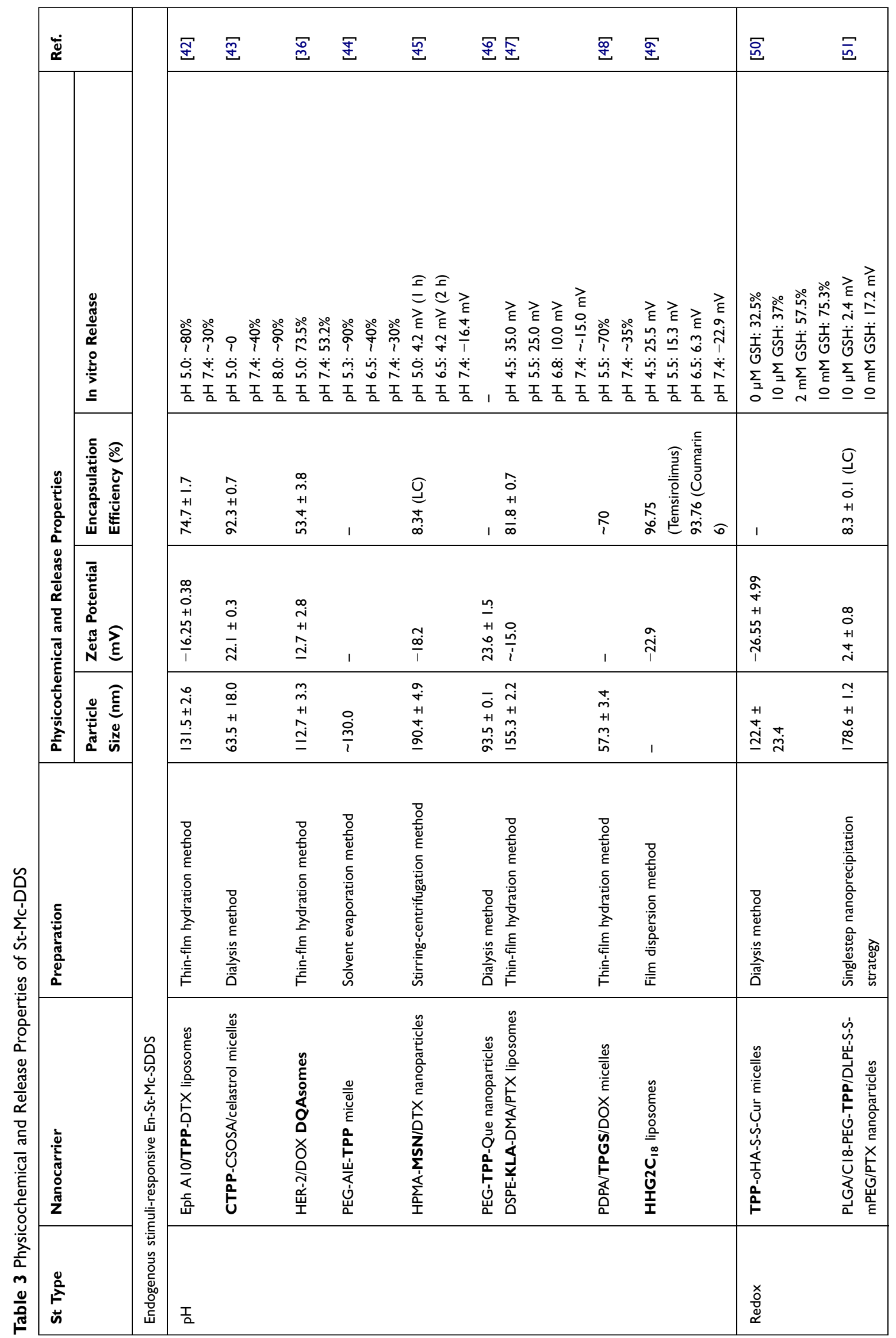




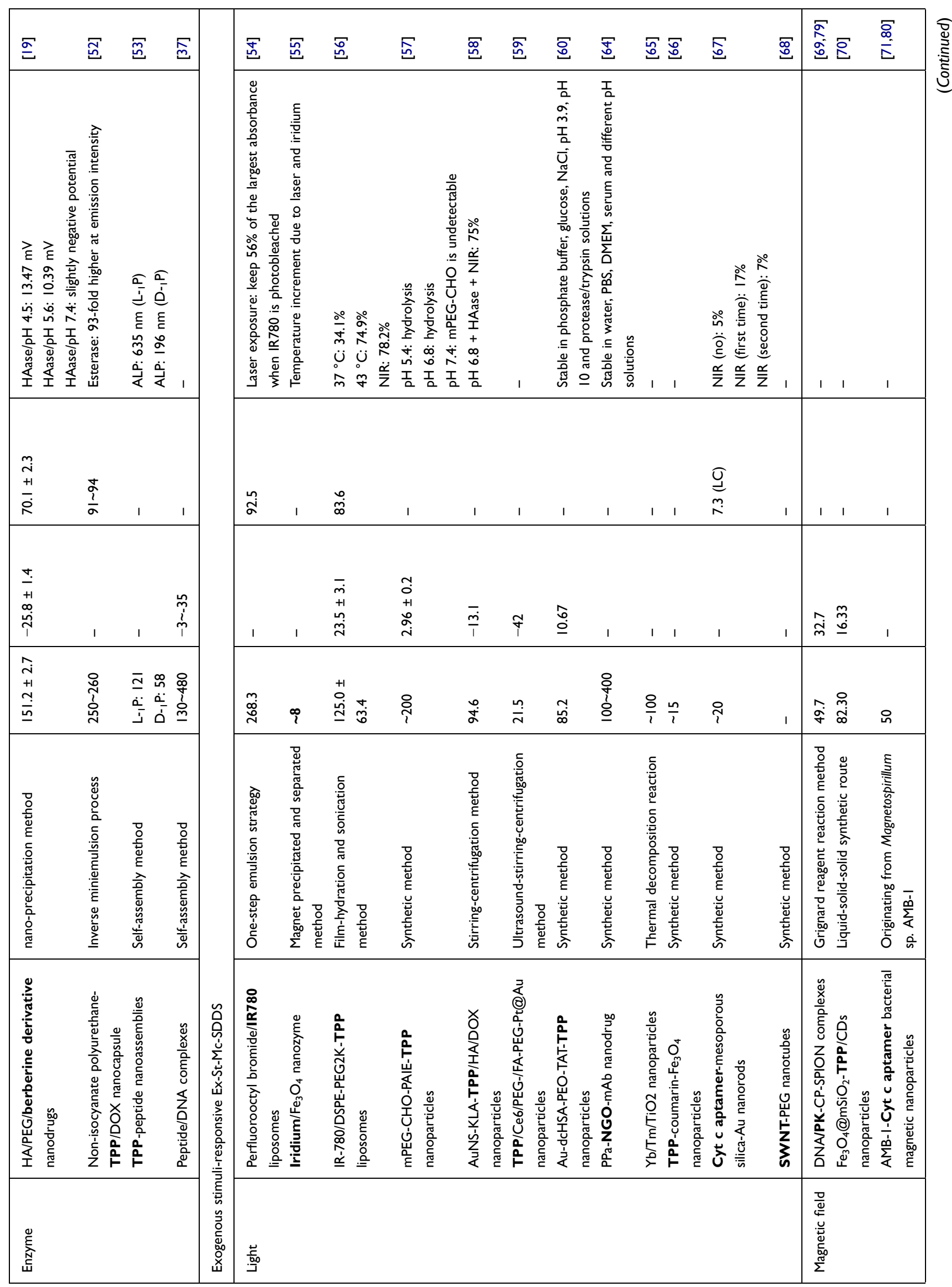




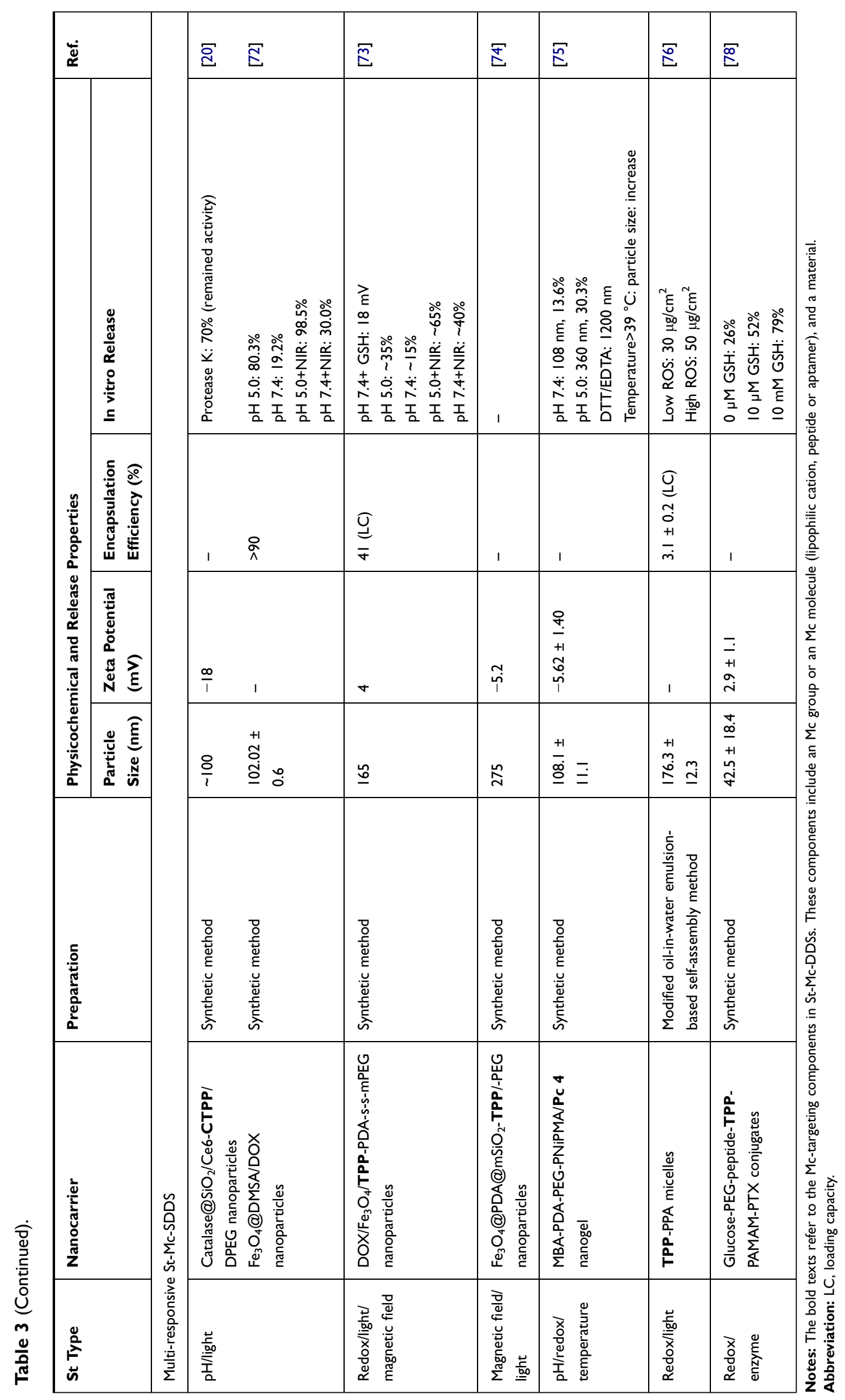




\begin{tabular}{|c|c|c|c|c|c|c|c|c|c|c|c|c|c|}
\hline$\stackrel{\check{\Phi}}{\ddot{x}}$ & $\overline{\bar{\infty}}$ & $\stackrel{\mathbb{g}}{\Psi}$ & $\stackrel{p}{\dot{P}}$ & 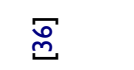 & 军异 & 疋 & 守 & 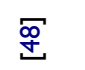 & 䇝 & 己्் & 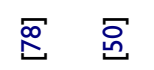 & $\bar{\Xi}$ & $\stackrel{5}{5}$ \\
\hline 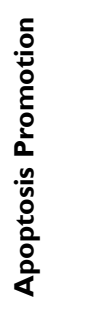 & 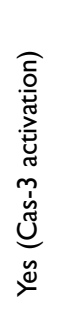 & 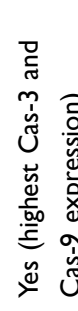 & 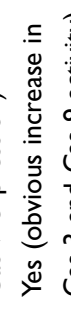 & 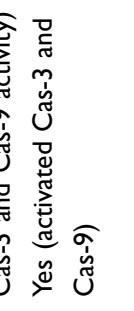 & $\stackrel{\mathscr{\varpi}}{\check{⿹}^{\mathscr{E}}}$ & 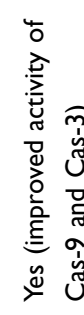 & 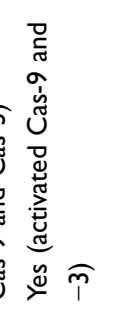 & $\overbrace{\nu}^{\mathscr{y}}$ & 1 & $\stackrel{\mathscr{y}}{\check{\nu}}$ & 1 & 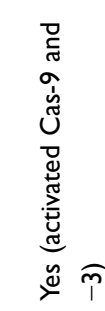 & 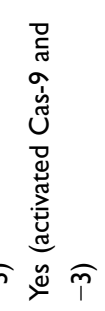 \\
\hline 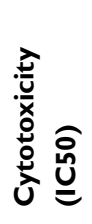 & 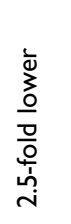 & 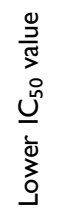 & 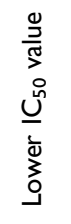 & 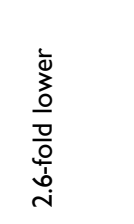 & 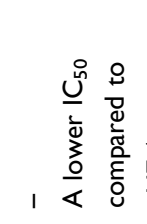 & 1 & 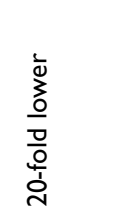 & 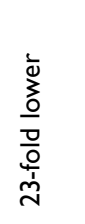 & 1 & 1 & 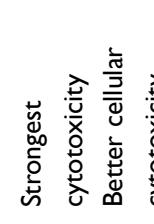 & 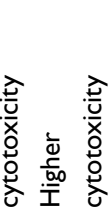 & 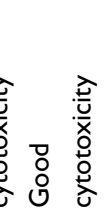 \\
\hline $\begin{array}{l}\widehat{\xi} \\
\frac{g}{2} \\
\frac{0}{\Sigma}\end{array}$ & 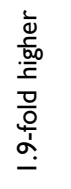 & 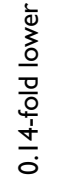 & 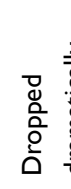 & 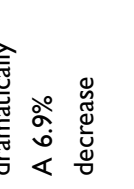 & $\begin{array}{l}\stackrel{0}{0} \\
\stackrel{0}{\overline{0}} \\
\end{array}$ & 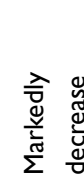 & 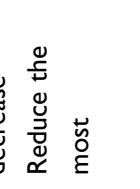 & 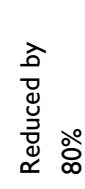 & 1 & 1 & 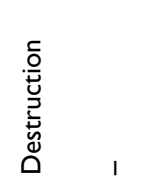 & 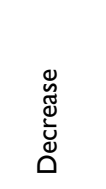 & 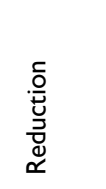 \\
\hline 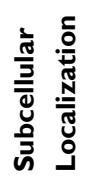 & 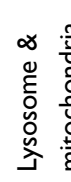 & 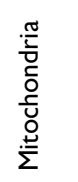 & 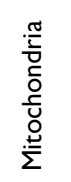 & 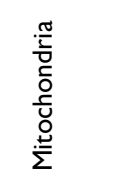 & 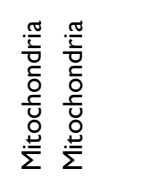 & 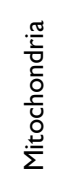 & 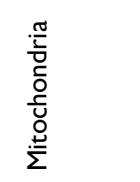 & 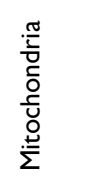 & 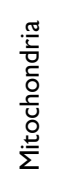 & 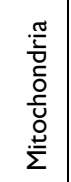 & 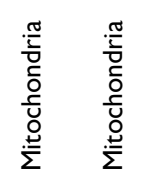 & 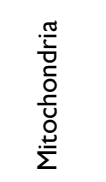 & 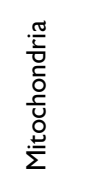 \\
\hline 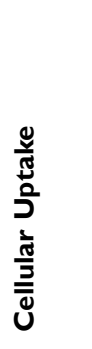 & 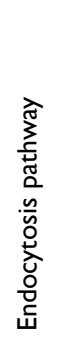 & 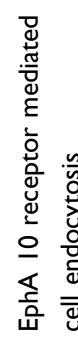 & 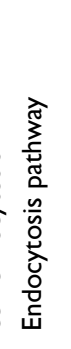 & 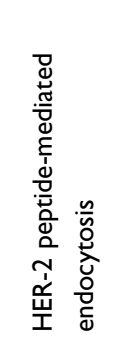 & 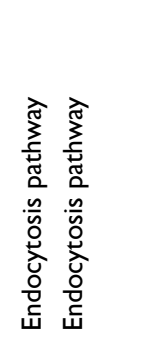 & 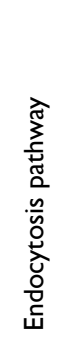 & 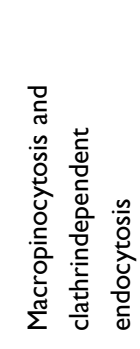 & 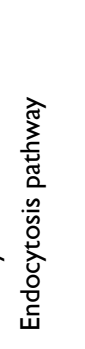 & 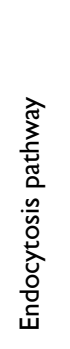 & 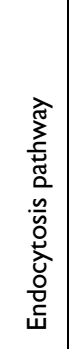 & 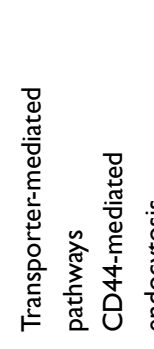 & 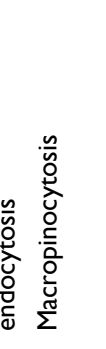 & 1 \\
\hline $\begin{array}{l}\stackrel{\mathscr{J}}{\Xi} \\
\overline{\bar{\Xi}}\end{array}$ & $\bar{F}$ & $\stackrel{\widehat{U}}{\stackrel{U}{U}}$ & $\begin{array}{c}\hat{U} \\
\stackrel{u}{\Sigma}\end{array}$ & 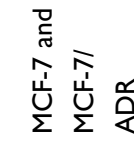 & 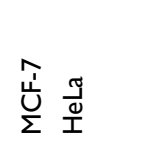 & $\frac{\widetilde{J}}{\underline{\Psi}}$ & 髉 & 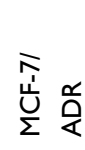 & 产 & $\bar{F}$ & 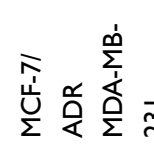 & $\bar{N}$ & 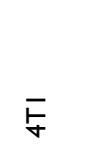 \\
\hline 总 & ○ั & 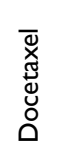 & 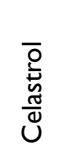 & 음 & 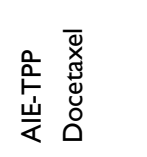 & 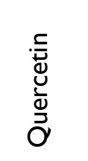 & 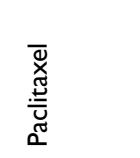 & ํㅡㅁ & \multicolumn{2}{|c|}{ 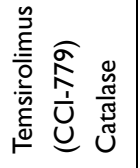 } & 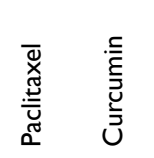 & 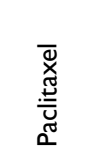 & 吕 \\
\hline 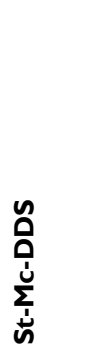 & 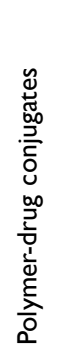 & 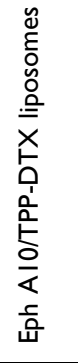 & 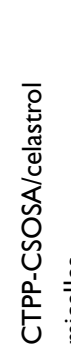 & 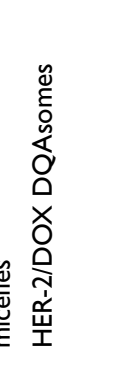 & 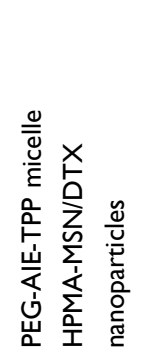 & 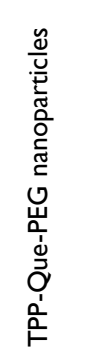 & 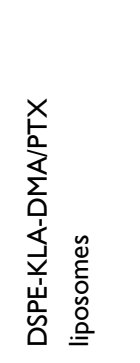 & 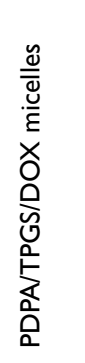 & 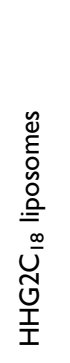 & 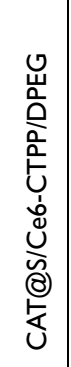 & 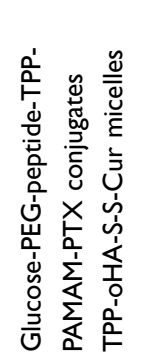 & 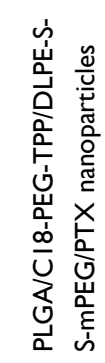 & 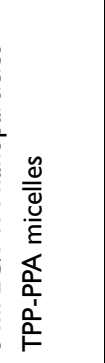 \\
\hline $\begin{array}{l}\stackrel{0}{2} \\
\ddot{z} \\
\ddot{z}\end{array}$ & $\frac{T}{a}$ & & & & & & & & & & $\begin{array}{l}\text { o } \\
\stackrel{0}{0} \\
\stackrel{\alpha}{\alpha}\end{array}$ & & \\
\hline
\end{tabular}




\begin{tabular}{|c|c|c|c|c|c|c|c|c|c|c|c|c|c|}
\hline$\ddot{\mathscr{\varpi}}$ & $\sigma$ & స్ర & 氙 & 壳 & 员 & ద్ర & 吕 & 呁 & 悉 & గ్ర్ర & 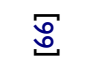 & ह్ & ब్ \\
\hline 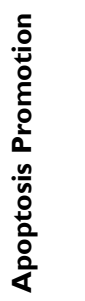 & 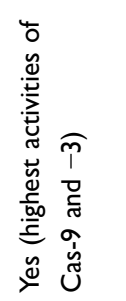 & 1 & $\stackrel{\check{\nu}}{\nu}$ & 1 & $\stackrel{\mathscr{y}}{\nu}$ & 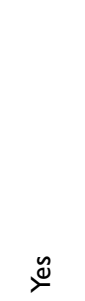 & $\stackrel{\check{\nu}}{\check{\nu}}$ & 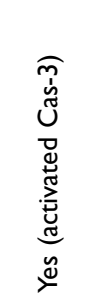 & $\stackrel{\check{\nu}}{\check{\nu}}$ & 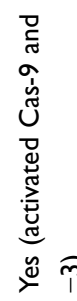 & $\hat{\imath} \stackrel{0}{\nu}$ & $\stackrel{\mathscr{\Perp}}{\succ}$ & $\stackrel{\check{0}}{\nu}$ \\
\hline 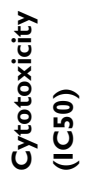 & 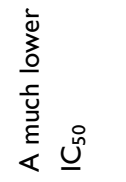 & I & 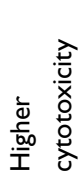 & 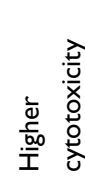 & 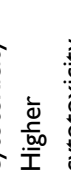 & 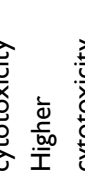 & 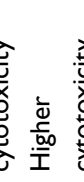 & 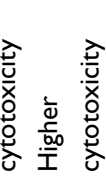 & 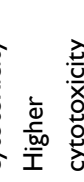 & 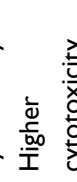 & 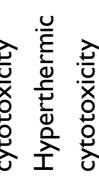 & 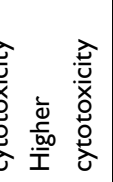 & 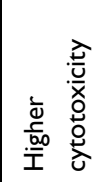 \\
\hline 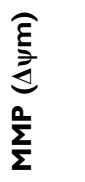 & 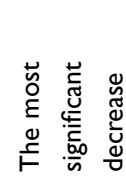 & 1 & 1 & 1 & 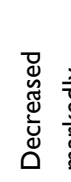 & 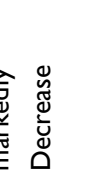 & 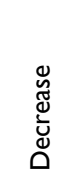 & 号 & 1 & 苟 & 1 & 1 & 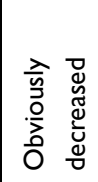 \\
\hline 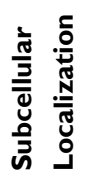 & 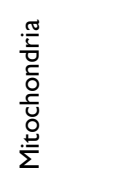 & 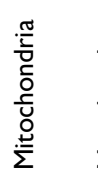 & 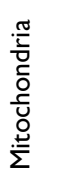 & 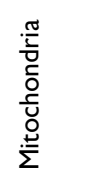 & 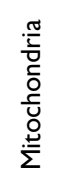 & 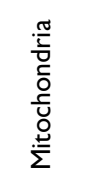 & 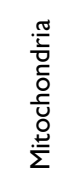 & 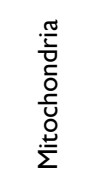 & 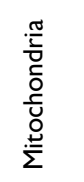 & 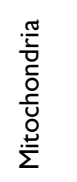 & 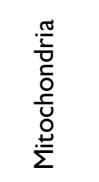 & 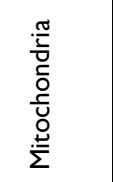 & 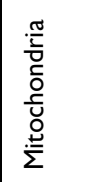 \\
\hline 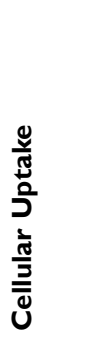 & 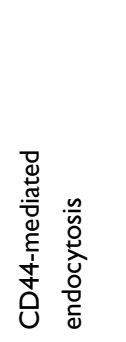 & । & 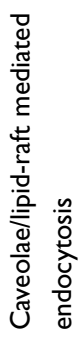 & 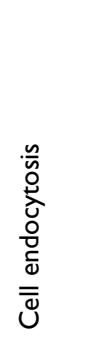 & 1 & 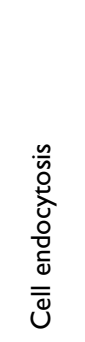 & 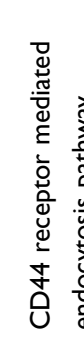 & 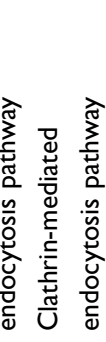 & 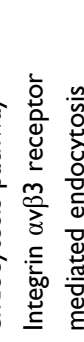 & । & 1 & 1 & 1 \\
\hline 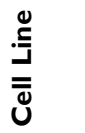 & 旁 & $\underset{\text { స్ }}{\text { Ż }}$ & $\begin{array}{l}\tilde{o} \\
\tilde{o} \\
\tilde{n}\end{array}$ & F & $\frac{\frac{\pi}{0}}{\frac{\pi}{I}}$ & $\Xi$ & ứ & $\begin{array}{l}\hat{U} \\
\frac{\hat{U}}{\Sigma}\end{array}$ & $\begin{array}{l}\text { U } \\
\sum_{i} \\
\text { D }\end{array}$ & $\frac{\frac{\pi}{0}}{I}$ & $\frac{\frac{\pi}{0}}{\frac{0}{I}}$ & $\frac{\frac{\pi}{0}}{I}$ & $\frac{\frac{\pi}{0}}{I}$ \\
\hline 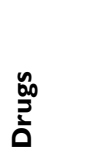 & 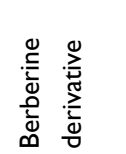 & ơ & 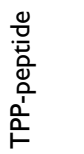 & 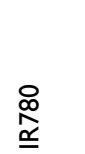 & $\begin{array}{l}\underline{\xi} \\
\stackrel{\underline{\Xi}}{\underline{\underline{\Xi}}}\end{array}$ & 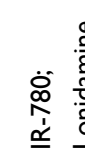 & 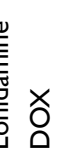 & Ů & $\frac{\pi}{\alpha}$ & $\frac{\stackrel{O}{E}}{\stackrel{i}{\varepsilon}}$ & 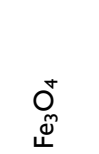 & 飞 & 衣 \\
\hline 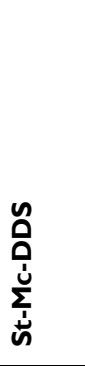 & 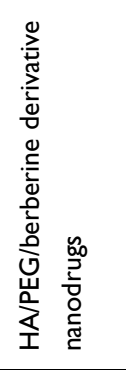 & 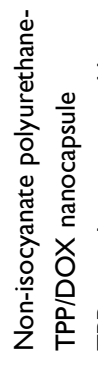 & 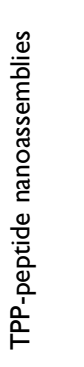 & 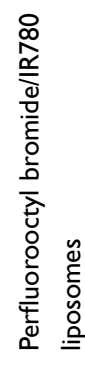 & 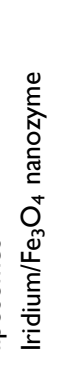 & 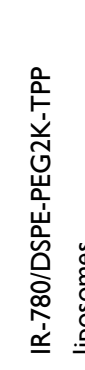 & 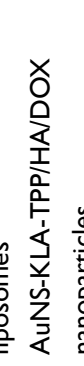 & 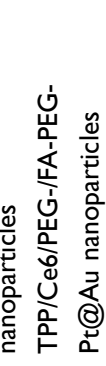 & 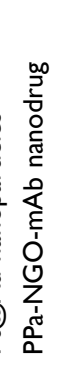 & 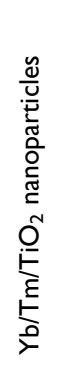 & 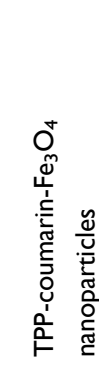 & 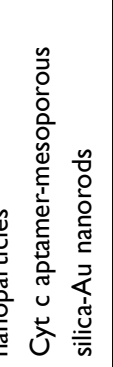 & 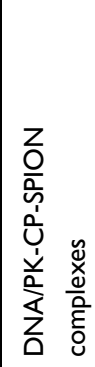 \\
\hline $\begin{array}{l}\stackrel{0}{\grave{z}} \\
\stackrel{\Delta}{\Delta}\end{array}$ & 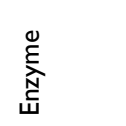 & & & 蕛 & & & & & & & & & 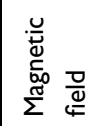 \\
\hline
\end{tabular}




\begin{tabular}{|c|c|c|c|c|c|c|c|c|c|c|}
\hline 远 & $\overline{\bar{\infty}} \quad \stackrel{\Xi}{\mathbb{S}}$ & $\stackrel{p}{\dot{g}}$ & ळ্ల & 声 & 阜 & 疋 & 导 & 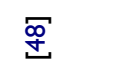 & $\frac{\sigma}{g}$ & 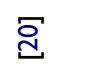 \\
\hline 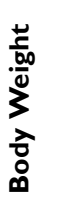 & 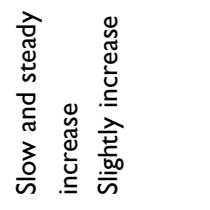 & 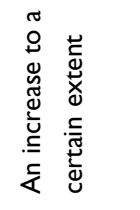 & 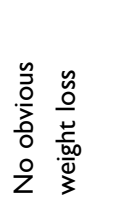 & 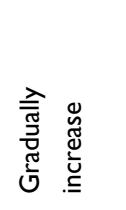 & 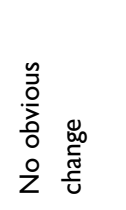 & 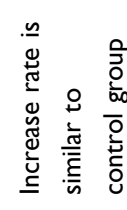 & 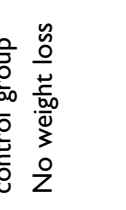 & 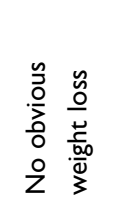 & 1 & 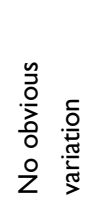 \\
\hline 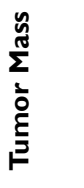 & 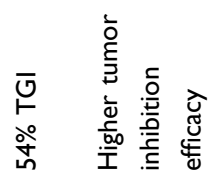 & $\begin{array}{l}\bar{\Xi} \\
\stackrel{2}{\circ} \\
\frac{\infty}{8}\end{array}$ & $\begin{array}{l}\text { ভ } \\
\stackrel{\circ}{\circ} \\
\stackrel{\leftrightarrow}{i}\end{array}$ & 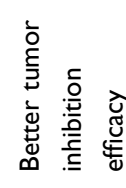 & 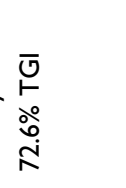 & $\begin{array}{l}\bar{v} \\
\stackrel{2}{\circ} \\
\stackrel{\hat{\circ}}{0}\end{array}$ & $\begin{array}{l}\bar{v} \\
\stackrel{\circ}{\circ} \\
\infty\end{array}$ & 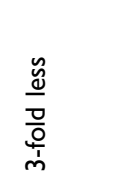 & 1 & 1 \\
\hline 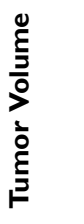 & 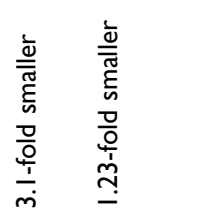 & $\begin{array}{l}\frac{\grave{\bar{\omega}}}{\overline{\bar{\sigma}}} \\
\underline{\omega}\end{array}$ & 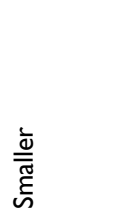 & 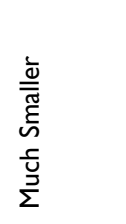 & 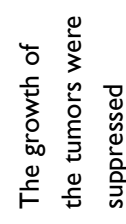 & $\frac{\grave{\varpi}}{\overline{\bar{\varpi}}}$ & 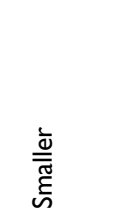 & 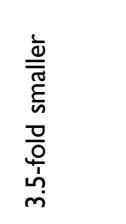 & 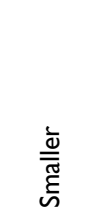 & 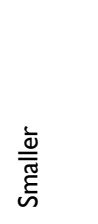 \\
\hline 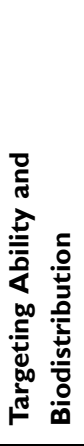 & 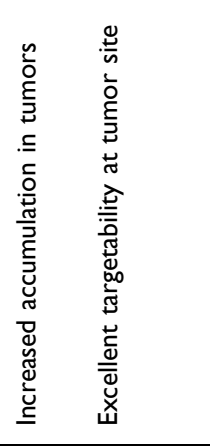 & 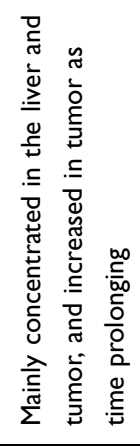 & & 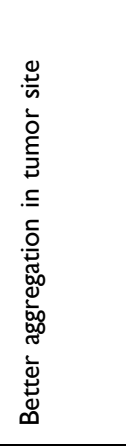 & 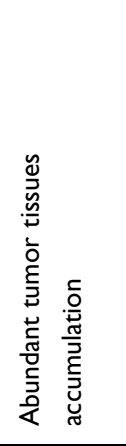 & 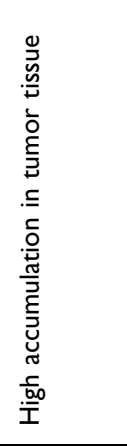 & 1 & 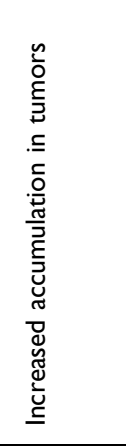 & 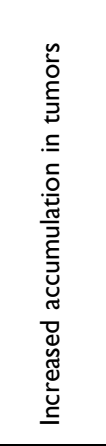 & 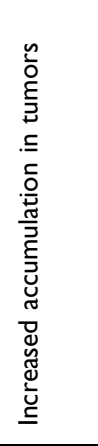 \\
\hline 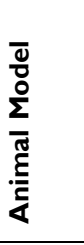 & 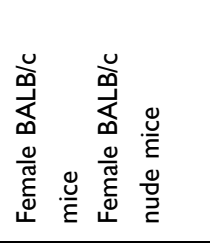 & 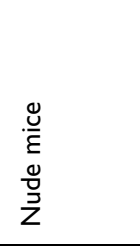 & 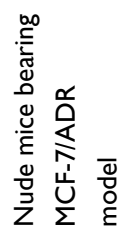 & 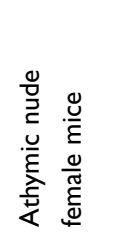 & 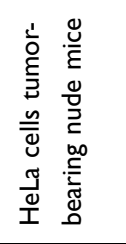 & 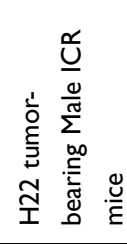 & 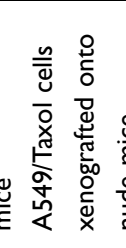 & 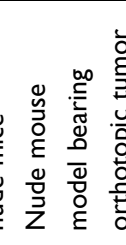 & 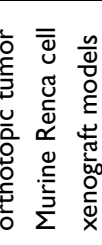 & 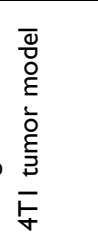 \\
\hline 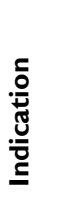 & 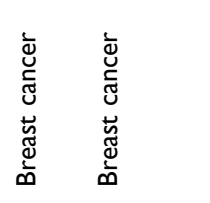 & 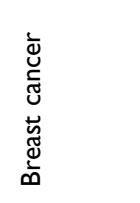 & 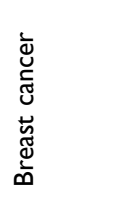 & 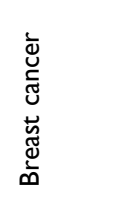 & 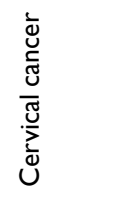 & 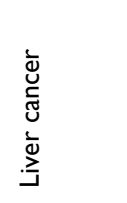 & 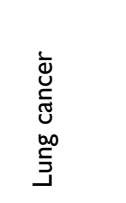 & 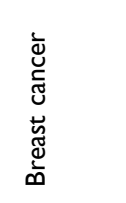 & 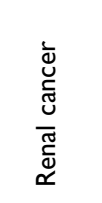 & 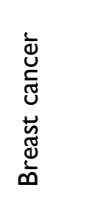 \\
\hline 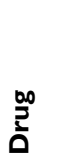 & 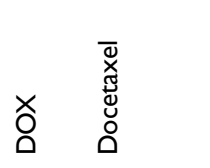 & 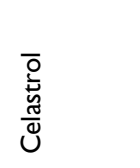 & $\stackrel{x}{\circ}$ & $\begin{array}{l}\stackrel{0}{\hat{K}} \\
\frac{\dot{w}}{\ll}\end{array}$ & $\begin{array}{l}\overline{\mathrm{J}} \\
\stackrel{\mathrm{J}}{\mathrm{U}} \\
\text { o }\end{array}$ & 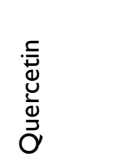 & 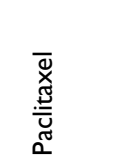 & ○̊ & 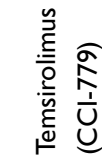 & U. \\
\hline 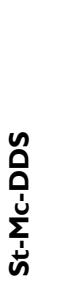 & 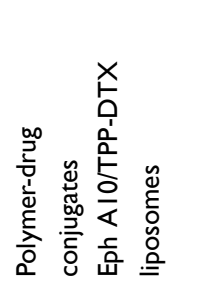 & 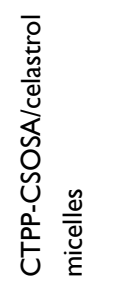 & 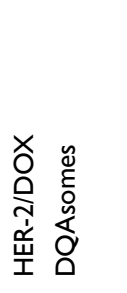 & 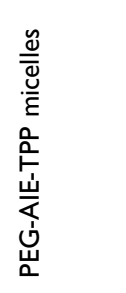 & 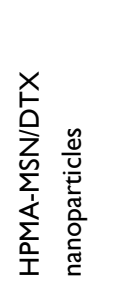 & 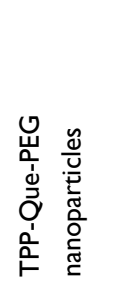 & 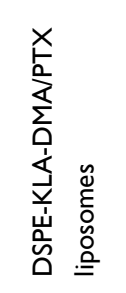 & 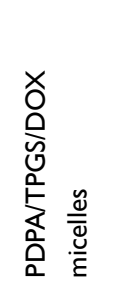 & 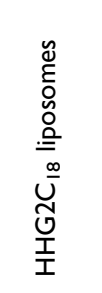 & 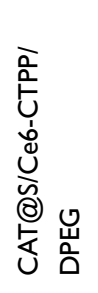 \\
\hline $\begin{array}{l}\stackrel{0}{2} \\
\stackrel{2}{z} \\
\ddot{n}\end{array}$ & $\frac{1}{a}$ & & & & & & & & & \\
\hline
\end{tabular}




\begin{tabular}{|c|c|c|c|c|c|c|}
\hline 迆 & $\stackrel{\infty}{=}$ & 문 & $\overline{\bar{n}} \quad \stackrel{D}{5}$ & 正 & $\sqrt[\widetilde{\Omega}]{2}$ & 氙 \\
\hline 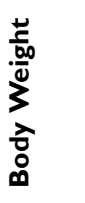 & 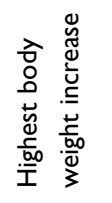 & & 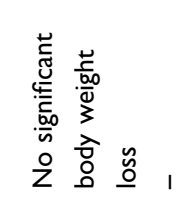 & \multicolumn{2}{|c|}{ 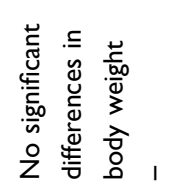 } & 1 \\
\hline 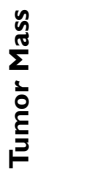 & 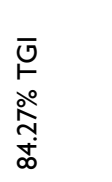 & 1 & $\begin{array}{l}\bar{J} \\
\vdash \\
\stackrel{\circ}{\hat{n}} \\
\stackrel{2}{\circ}\end{array}$ & 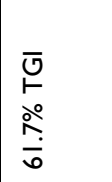 & 1 & 1 \\
\hline 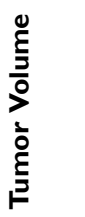 & 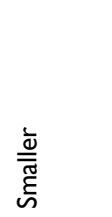 & । & 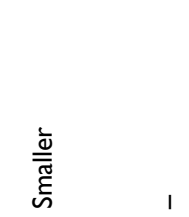 & 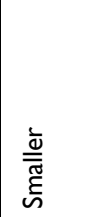 & 1 & 1 \\
\hline 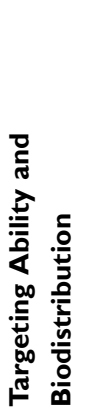 & 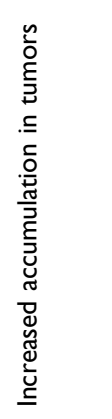 & 1 & 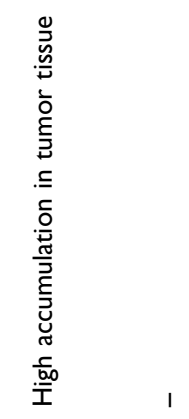 & 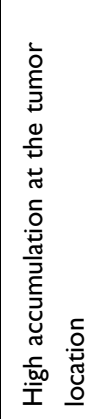 & 1 & 1 \\
\hline 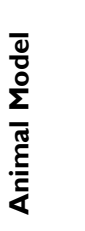 & 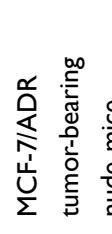 & 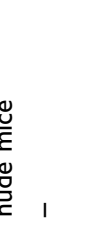 & 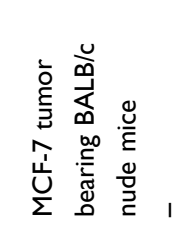 & 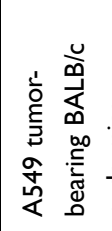 & 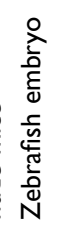 & 1 \\
\hline 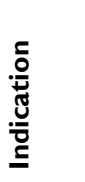 & 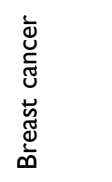 & 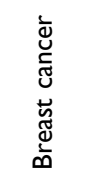 & 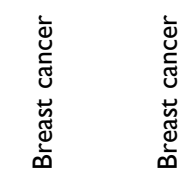 & 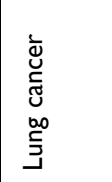 & 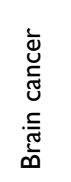 & 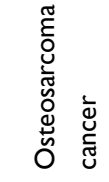 \\
\hline 足 & 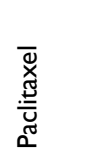 & 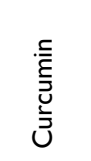 & 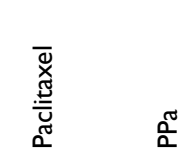 & 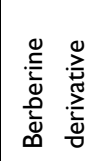 & ○̊ & 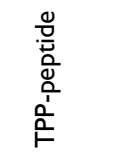 \\
\hline 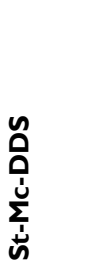 & 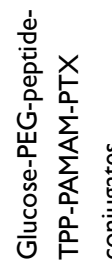 & 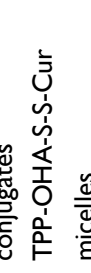 & 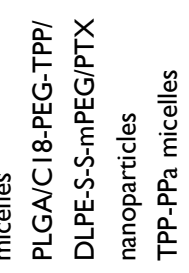 & 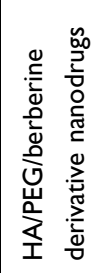 & 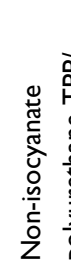 & 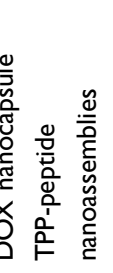 \\
\hline 离 & $\begin{array}{l}\text { ô } \\
\stackrel{o}{\ddot{d}} \\
\propto\end{array}$ & & & 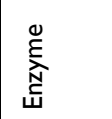 & & \\
\hline
\end{tabular}




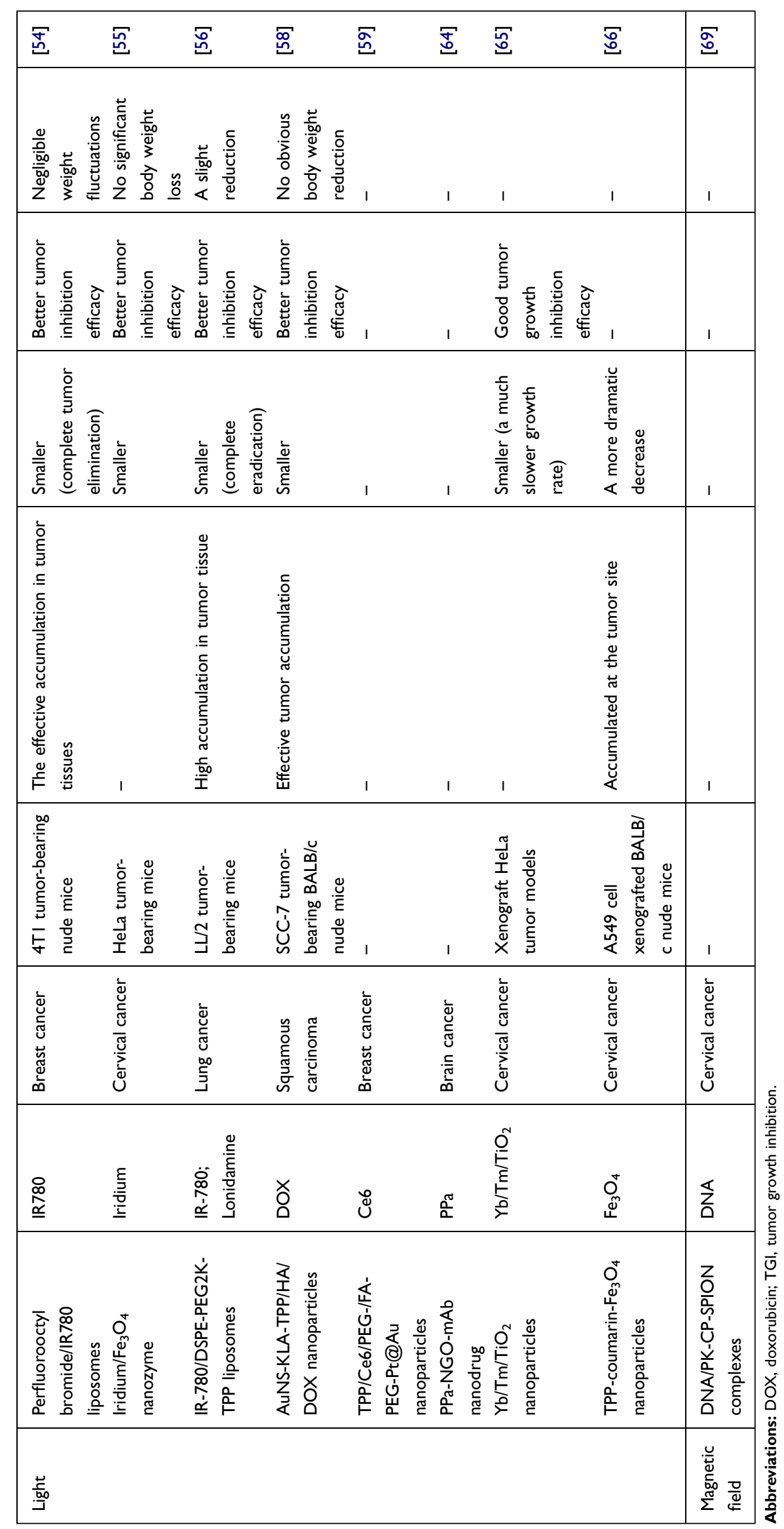


to the Mc. The SS peptide is taken up by the cell and inner Mc membrane by an energy-independent mechanism. ${ }^{5}$ In order to meet different investigative requirements, MPPs are designed to have different functions and structures (eg, different amino acid sequences, hydrophobicities and peptide charges). For example, incorporating the D-isomer of arginine into MPPs protects the peptides from enzymatic degradation, ${ }^{34,101}$ integrating the targeting motif for cell ingestion and the proapoptotic motif into MPPs for enhanced anticancer effects. ${ }^{10,102,103}$

The MQ peptide sequence is an Mc-targeting sequence (MTS, also called an Mc signal peptide). ${ }^{37}$ MTSs usually consist of 20 30 amino acids, which reach the Mc through the use of a translocase from the outer and inner membrane complexes by mimicking the cellular mechanism of Mc protein delivery. ${ }^{34,104,105}$ That is, MTSs create chimeric proteins and are taken up via the Mc protein import machinery. ${ }^{5}$ The multifunctional peptide/DNA complex facilitates DNA targeting to the $\mathrm{Mc} .^{37}$ In a peptide chain having lysine-histidine fragment, cation contributes to cross cellular membrane, lysine condenses DNA for cellular ingestion, and histidine helps DNA to reach cytosol by endosomal lysis through proton sponge effect. Once this type of peptide is attached to a Mc-specific peptide sequence, it will effectively facilitates the targeting of DNA to the Mc.

However, the deep mechanism of Mc-targeting characteristics of peptides is unclear. In contrast to hydrophobic cations, peptides are degradable and cause slight cytotoxicity after Mc deposition. Peptides have large molecular weights, so they might induce undesired immune responses; peptide degradation might cause Mc-targeting failure; ${ }^{106}$ some peptides are difficult to synthesize or to be conjugated with targeting materials; and the characteristics and abilities of some targeting materials conjugated with Mc-targeting peptides might be affected. ${ }^{107}$

\section{Mc Aptamer-Based St-Mc-DDSs}

The Mc cytochrome (Cyt) C aptamer ${ }^{67,71}$ is employed to make St-Mc-DDSs efficiently accumulate in the Mc of cancer cells. The Cyt $\mathrm{C}$ aptamer is a short single-stranded oligonucleotide sequence that specifically recognizes $\mathrm{CytC}^{108,109}$ which is normally located in the inner $\mathrm{Mc}$ membrane and plays a key role in ATP synthesis. ${ }^{67,71}$ Cyt $\mathrm{C}$ is secreted into the cytosol and induces apoptosis through a Mc pathway. ${ }^{59,110,111} \mathrm{Cyt} \mathrm{C}$ aptamers have been attached on the outside of smart mesoporous silica-encapsulated gold nanorods ${ }^{67}$ and bacterial magnetic nanoparticles ${ }^{71}$ to allow them to efficiently accumulate in the Mc of cancer cells. Mc-targeting aptamers are a prospective strategy for St-Mc-DDSs. Compared to peptides, aptamers are much easier to synthesize while harder to be biodegraded and denatured by further modification. Aptamer has large molecule, so its conjugation efficiency with targeting material is low and targeting efficiency is probably influenced. Aptamer is so expensive that the clinical application of aptamer-based St-Mc-DDSs is severely hindered. ${ }^{107}$

In addition to Cyt $\mathrm{C}$ aptamers, other Mc-targeted aptamers have been applied. An aptamer containing folding guanine-rich RNA/DNA was designed to modify nanocomplexes for Mc imaging and decrease the Mc membrane potential. ${ }^{112}$ A dual-ligand liposomal system was decorated with a Mc RNA aptamer (RNase P) that enhanced cellular uptake and achieved Mc targeting. ${ }^{113}$ The short RNA aptamer Mitomer 2 showed good binding affinity to the Mc and resistance to degradation by nucleases. ${ }^{114}$

\section{Mc Materials-Based St-Mc-DDSs}

Mc materials such as colloidal dequalinium vesicles ${ }^{36}$ and carbon nanomaterials ${ }^{64,68,70,115,116}$ have been used in StMc-DDSs. Amphiphilic dicationic dequalinium may selfassemble to form aggregates DQAsomes. ${ }^{5}$ DQAsomes display a positive surface charge in aqueous environments and accumulate in the Mc in response to the electrochemical gradient across the $\mathrm{Mc}$ membrane system. ${ }^{36}$ DQAsomes serve as a mitochondriotropic carriers to deliver hydrophobic drugs and genes to the Mc. ${ }^{117,118}$

Carbon-based nanomaterials (such as graphene, carbon dots and carbon nanotubes) have been emerging as new biomaterials to design and fabricate St-Mc-DDSs due to their high tunability, good biocompatibility and other unique physicochemical characteristics. ${ }^{64,68,70,116,119,120}$ They selectively target the Mc based on their polarization and positive charge. In graphene oxide (NGO)-based StMc-DDSs, graphene serves not only as a carrier material for Mc targeting but also as a phototherapy agent. ${ }^{64}$ The hypericin-functionalized NGO nanoparticles enhance Mctargeting and the synergistic anticancer effects of phototherapy and chemotherapy. ${ }^{115}$ A novel type of fluorescent carbon dot achieved Mc imaging and Mc-targeted PDT without further modification by other mitochondriotropic ligands (such as TPP). ${ }^{116} \mathrm{~A}$ dual Mc-targeting moiety (TPP and carbon dot)-modified biocompatible platform (magnetic mesoporous silica nanovesicles) achieved long-term imaging and magnetic field-enhanced cellular 
uptake. $^{70}$ Mc-targeting single-walled carbon nanotubes (SWCNs) are used for cancer photothermal therapy. ${ }^{68}$

\section{Stimuli-Responsive Characteristics of St-Mc-DDSs}

\section{Endogenous Stimuli-Responsive St-Mc- DDSs}

En stimuli-responsive St and Mc-targeted platforms (En/ St-Mc-DDS) have gradually been used to enhance cancer treatment efficacy. En stimuli in the cancerous microenvironment provide signals for anticancer DDSs to accumulate in tumor tissues/cells and release drugs in an on-demand manner. ${ }^{119}$ The physiological signals facilitating tumor targeting include an acidic media, ${ }^{121}$ overexpressed enzymes (such as matrix metalloproteinase, cathepsin, phospholipase, and oxidoreductase) ${ }^{122}$ or membrane-protein makers, ${ }^{123}$ ATP, ${ }^{124}$ intracellular glutathione $(\mathrm{GSH})^{125}$ or hypoxic features. ${ }^{126}$ According to the En/St type, ${ }^{127,128}$ $\mathrm{En} / \mathrm{St}-\mathrm{Mc}-\mathrm{DDSs}$ are mainly classified into three types ( $\mathrm{pH}-$ , redox- and enzyme-responsive St-Mc-DDSs). Their recent advances are described as follows. To date, there have been no reports of En (other biomolecules outside of our list) stimuli-responsive St-Mc-DDSs.

\section{$\mathrm{pH}-$ Responsive St-Mc-DDSs}

Compared to normal tissues, tumors have higher metabolism and most of them have lower $\mathrm{pH}$ values of extracellular and intracellular fluids $(6.5 \sim 7.0 \mathrm{vs} \sim 7.4 ; 5.0 \sim 6.0 \mathrm{vs} \sim 7.2) .{ }^{129}$ The organelles of tumor cells such as Mc $(\sim 8.0),{ }^{43,130,131}$ lysosomes (4.5 5.0), endosomes (5.5 6.0) and cytosol $(7.4)^{132}$ have different $\mathrm{pH}$ values. Therefore, Mc-targeted nanocarriers with a particular responsiveness to $\mathrm{pH}$ can intently release drugs at tumor locations, simultaneously preventing the unwanted release in normal tissue. Weakly acidic/basic compounds are suitable constituents for the preparation of $\mathrm{pH}$-responsive Mc-targeted platforms. For example, compounds containing $-\mathrm{COOH},-\mathrm{NH}_{2}$ or $-\mathrm{SO}_{3} \mathrm{H}$ groups may alter from their neutral to ionized forms, ${ }^{133}$ and further induces dramatic alteration in the interaction or affinity between the drug molecule and drug vesicle. The $\mathrm{pH}$ gradients are stimuli that release drug from $\mathrm{pH}$-sensitive Mctargeted systems. $^{36,42-49}$

Two easy measures have been employed to design $\mathrm{pH}-$ responsive St-Mc-DDSs. ${ }^{134}$ The first is based on acidcleavable linkers such as a Schiff-base, hydrazone, acetal/ketal, amide or cis-aconityl. The second is based on the degradation of the polymer and destabilization of the nanocarrier in a $\mathrm{pH}$-sensitive manner. $\mathrm{pH}$-sensitive StMc-DDSs usually have the following functional features: they expose the carrier core or overturn the positive charge in the tumor extracellular environment to promote carrier uptake and they degrade the carrier inside the cells to achieve rapid drug release or proton sponge action to promote endosome escape.

The polyethylene glycol (PEG)-Schiff base-cholesterol derivate was synthesized and attached to the liposomes (PSLP). ${ }^{42}$ Schiff base bond is hydrolyzed in acidic media, and the PEG shell is removed from the liposomes. The remaining lipophilic PSLP is exposed and easily internalized by tumor cells. Dioleoyl phosphoethanolamine (DOPE) is a constituent of lamellar PSLP that exists in a hexagonal phase at physiological $\mathrm{pH}$ but is deformed in acidic medium. After internalization, DOPE merges with the lysosomal membrane and releases the drugs into the cytosol. Then, the drug accumulates in Mc through the guidance of TPP. In addition to the combination of stimulitriggered St- and Mc-targeted strategies, other active targeted strategies (such as Eph receptor A10 (EphA 10)mediated cellular endocytosis) and passive targeted strategies (such as the enhanced permeability and retention effect, EPR) have been applied simultaneously to achieve better St-Mc delivery. ${ }^{42}$

A pH-responsive St-Mc nanohybrid comprised a N-(2hydroxypropyl) methacrylamide (HPMA) copolymer shell and a positively charged nanovesicle core was fabricated via electrostatic interactions. ${ }^{45}$ Under mildly acidic environment of the tumor, the first-stage $\mathrm{pH}$-responsiveness took place when the hydrolysis of the amide bonds in the HPMA copolymers occurred and the charge of the copolymer changed from negative to positive, which was beneficial for cellular ingestion. The second-stage occurred in endosomes/lysosomes due to the proton sponge effect, which facilitated Mc location.

Another $\mathrm{pH}$-responsive St-Mc-DDS was prepared to induce cellular apoptosis. ${ }^{47}$ This liposome contains a hybrid lipid by synthesizing Mc peptide KLA with dimethylmaleic anhydride via amide bond and 1,2-distearoyl-sn-glycero-3-phosphoethanolamine (DSPE). This liposome exhibited positive charge at $\mathrm{pH} \sim 6.8$ of extracellular media to facilitate its entrance into cancerous cells. After that, KLA purposely delivered cargo to Mc.

An alkaline pH-responsive St-Mc-DDS was constructed. $^{43}$ Lipophilic CTPP conjugated with glucolipid-like conjugates formed micelles in aqueous solution and encapsulated celastrol in the hydrophobic core. These 
micelles selectively responded to the Mc alkaline $\mathrm{pH}(\mathrm{pH}$ 8.0), while reduced drug leakage occurred in the cytoplasm ( $\mathrm{pH}$ 7.4) and lysosomes ( $\mathrm{pH}$ 5.0). The acidity/basicity of the loaded drug was relevant to the drug release rate and solubility in different environments.

\section{Redox-Responsive St-Mc-DDSs}

The redox/oxidation states between the intracellular and extracellular matrices of tumors are very different. For example, the GSH level in the cytosol $(2-10 \mathrm{mM})$ of tumor cells is $\sim 1000$ times greater than that in the extracellular matrix $(2-20 \mu \mathrm{M})$ or $>4$ times greater than that in the normal cells, which renders the tumor intracellular redox potential. ${ }^{135}$ Redox-responsive functional bonds or groups $^{8}$ such as disulfide, phenylboronic acid and ester bonds, and singlet oxygen-responsive imidazoles have been used to design redox-responsive St-Mc-DDSs.

Disulfide bonds are incorporated into either the crosslinker or polymer structure of DDSs. Disulfide bonds are converted to thiol groups by various intracellular stimuli, such as the reducing agent $\mathrm{GSH}^{8}{ }^{8} \mathrm{~A} \mathrm{Sm}-$ Mc-DDS for overcoming multidrug resistance (MDR) had a dendrimer core co-decorated with paclitaxel (PTX) via a disulfide bond and TPP via an amido bond, and a shell covered with PEG layer via a MMP2-sensitive peptide. $^{78}$ Once the core detached from the PEG layer, it targeted the Mc via TPP guidance, and PTX was rapidly released through a reductive reaction. Another Sm-Mc-DDS was TPP-oligomeric hyaluronic acid-S-S-curcumin-loaded micelles. ${ }^{50}$

Phenylboronic acid and ester bonds are sensitive to $\mathrm{H}_{2} \mathrm{O}_{2}$. Arylboronic esters and thioketal linkers are oxidized by $\mathrm{H}_{2} \mathrm{O}_{2}$ at $50 \mu \mathrm{M}^{136}$ and $100 \mu \mathrm{M},{ }^{137}$ respectively. The lipophilic neutral ferrocene/lipophobic cation ferrocenium redox pair was utilized for the design of cancerspecific, Mc-targeting moieties to trigger reactive oxygen species (ROS)-mediated drug release. ${ }^{138}$

A polymeric micelle with an imidazole group is singlet oxygen-responsive and able to deliver pyropheophorbide A (PPA)-TPP (a photosensitizer). ${ }^{76}$ The imidazole moiety was oxidized to hydrophilic urea upon triggering with light. The amphiphilicity of micelles changed, followed by rapid photosensitizer release and $\mathrm{Mc}$ inhibition via TPP. The PDT efficacy was then enhanced.

Generally, these redox-responsive St-Mc-DDSs disassemblied and released drug in response to ROS, through lipophobic-lipophilic transition or cleavage of ROSresponsive linkers. The high sensitivity and specificity have been confirmed by the above St-Mc-DDSs. However, there are still some challenges to be addressed: the degraded linkers should be histobiocompatible, nontoxic and non-immunogenic. Otherwise, they may lead to unwanted side effects and a varied redox state inside tumor cells, which is associated with phenylboronic acid and ester bonds. Due to the ROS dynamic and heterogeneity of tumor cells, it is difficult to control redox balance and understand related molecular mechanism. ${ }^{139}$

\section{Enzyme-Responsive St-Mc-DDSs}

Enzymes such as esterases, hyaluronidases (HAases) and alkaline phosphatases (APases) are concentrated inside the cellular cytoplasm or lysosomes or overexpressed in the extracellular environment of tumor sites. ${ }^{140}$ Catalytic reactions refer to the cleavage/formation of chemical bonds or the oxidation/reduction of substrates. Enzymatic activation ${ }^{141}$ using different enzymes as stimuli has been applied to design enzyme-responsive St-Mc-DDSs.

A new stable and monodispersing nonisocyanate polyurethane nanocapsule (NIPU) are developed. ${ }^{52}$ Their shells had a polyurethane-based ${ }^{142}$ polymeric backbone with embedded ester linkages in response to esterases. Their core loaded the hydrophilic drug doxorubicin (DOX) during the polymer synthesis and NIPU preparation process. NIPU was further post-grafted with phosphonium ions to achieve Mc-targeted release of the drug. Song et al ${ }^{19}$ first designed a Mc-targeted nanodrug (a positively charged 9O-octadecyl substituted berberine derivative, $\mathrm{BD}$ ) that was dually modified with DSPE-PEG2000 to increase the stability and the negatively charged hyaluronic acid to achieve tumor targeting and lysosomal escape through recognition by HAase in tumor tissue and lysosomes. TPP was attached to phosphorylated tetrapeptide enantiomers to obtain oligomers that would self-assemble to form nanosized assemblies in response to APase enzyme-catalyzed reaction. ${ }^{53}$ These assemblies further caused Mc dysfunction and killed cancer cells while minimizing acquired drug resistance.

In summary, enzymes as stimuli of St-Mc-DDSs have intrinsic merits: as endogenous components, they have inherent biocompatibility and biosafety; they have extraordinary selectivity for substrates and high catalytic efficiency; and the same enzyme family, such as matrix metalloproteinases, in tumor cells usually have similar active pockets that may lead to similar substrate preferences. $^{143}$ 


\section{Exogenous Stimuli-Responsive St-Mc- DDSs}

Ex stimuli such as magnetism and light have been employed to control drug release within a Mc-DDS (Ex/ Sm-Mc-DDS). Compared to En stimuli, Ex stimuli seem to have been more successful in overcoming the individual variability of controlled drug release. ${ }^{139,144}$ We will discuss the rationales and applications of light and magnetic responsive Ex/St-Mc-DDS. To date, the combination of other Ex stimuli (temperature, ultrasound, electric pulse, etc.) and Mc-DDS has not been reported.

\section{Light-Responsive St-Mc-DDS}

UV $(200 \sim 400 \mathrm{~nm})$ light only has weak penetrating power, and it is harmful to normal cells and tissues. In contrast, NIR light $(650 \sim 900 \mathrm{~nm})$ has better tissue penetration and an improved safety profile. ${ }^{145}$ Therefore, NIR-responsive DDSs with good spatiotemporal control are potential nanocarriers for practical therapy. Based on the NIRresponsive mechanism, there are three types of lightresponsive St-Mc-DDS: NIR light-activated PDT-, PTTand PDT/PTT-based St-Mc-DDSs.

Wei et $\mathrm{al}^{64}$ used NGO as a smart carrier, which was dually modified with a monoclonal antibody (mAb) for $\alpha v \beta 3$-positive tumor cell location and a PPA-PEG conjugate for phototoxicity in the organic environment of the Mc. The nanodrug PPA-NGO-mAb significantly enhanced Mc-mediated PDT apoptosis by yielding ROS such as singlet oxygen and free radicals. Hou et $\mathrm{al}^{65}$ designed titanium dioxide $\left(\mathrm{TiO}_{2}\right.$, shell)-decorated upconversion nanosystems. ${ }^{57}$ These core/shell nanocomposites (inorganic crystalline nanoparticles, 1-100 nm) transformed NIR light to UV emission, which triggered the cytotoxicity of $\mathrm{TiO}_{2}$. Therefore, under NIR irradiation, these nanocomposites served as an effective photosensitizer and generated intracellular ROS in the Mc to kill tumor cells.

PTT usually uses a photothermal conversion agent to convert NIR light into thermal energy for hyperthermia in the tumor region. Chen et al coencapsulated Mc-targeting gold nanostars (AuNSs) and DOX in a hyaluronic acid protective shell to fabricate a St-Mc-DDS, which was ingested into cancer cells upon recognition by CD44 receptor. DOX was then released for chemotherapy. The AuNSs codecorated with the cationic peptide R8 and proapoptotic peptide TPP-KLA acted as a Mc-targeting nanoheater for NIR-triggered PTT. ${ }^{58}$ Kong et al ${ }^{146}$ designed a microhybrid with two-photon absorption characteristics through coordination interactions between silver and a fluorescent cyano- carboxylic acid derivative. The decreased quantum fluorescence and improved two-photon absorption caused by the surface plasmon resonance effect led to good photothermal output in Mc of HeLa cancer cells when radiation at $780 \mathrm{~nm}$.

Zhang et al $^{54}$ designed a nanosystem by integrating IR780 into perfluorooctyl bromide (PFOB)-based nanoliposomes for synergistic PDT/PTT under NIR irradiation at 808 $\mathrm{nm}$. Mc-targeting IR780 is easily to be encapsulated into nanoliposomes due to its hydrophobicity. IR780 had PTT/ PDT effects, and the PDT effect was enhanced by the oxygen carried by PFOB. This Mc-targeting nanoliposome was better than the one consisting of indocyanine green (ICG) and PFOB. ${ }^{147}$ The latter had PTT/PDT effects but did not have a Mc-targeting effect. Luo et al ${ }^{148}$ synthesized a Mc-targeted NIR photosensitizer for jointly PTT/PDT by modifying heptamethine cyanine dye with different side-chain $\mathrm{N}$-alkyl.

In summary, in order to conquer resistance to chemotherapy, PTT and PDT are often applied jointly in a NIR-responsive St-Mc-DDS to achieve synergistic antitumor effects. However, the biocompatibility and biodegradability of the photosensitizer (especially inorganic nanoparticles) used in such delivery systems must be considered for clinical implications. ${ }^{149}$ In addition, the lightresponsive St-Mc-DDS is only suitable for the treatment of superficial tumors such as skin surface cancer and breast cancer due to limited light penetration.

\section{Magnetic Field-Responsive St-Mc-DDSs}

The application of magnetic materials along with external magnetic fields was first introduced to medicine by Freeman et $\mathrm{al}^{150}$ in 1960. Magnetic stimuli-triggered StDDSs features advantages over chemotherapy: ${ }^{151}$ they are a noninvasive approach to control drug release; ${ }^{152}$ they scarcely have any physical interaction with the body and are effective over a distance as long as a few centimeters; the nanocarriers can overcome blood flow resistance and arrive at the tumor region under the influence of a magnetic field spatially focused at desired sites; and upon removal of the external magnetic field, there is no residual magnetism or drug effects. Magnetic field-responsive St-Mc-DDSs usually use superparamagnetic iron oxide nanoparticles (SPIONs) $10 \sim 20 \mathrm{~nm}$ in size. Surface functionalization of SPIONs may overcome their drawbacks, such as a short blood circulation time due to aggregation and oxidization.

Kim et $\mathrm{al}^{69}$ modified SPIONs with PK11195 $\left(\mathrm{C}_{21} \mathrm{H}_{21} \mathrm{ClN}_{2} \mathrm{O}\right)$ and chitosan-graft-PEI to fabricate a Mctargeting gene carrier, which effectively condensed and protected DNA. Under an exterior magnetic field, the 
transfection productivity of this gene carrier was comparable to PEI 25 K. PK11195 facilitated the accumulation in the Mc and activated apoptosis. This magnetofection of the magnetic-responsive St-Mc-DDS led to an enhanced therapeutic effect on tumor cells. Zhang et al ${ }^{70}$ constructed a Mc-targeting nanoplatform of iron oxide silica nanovesicles decorated with TPP. The cellular uptake efficiency of the nanoplatform was enhanced upon application of a magnetic field of $0.30 \mathrm{~T}$. SPIONs increased temperature under magnism to kill tumor cells by hyperthermia. ${ }^{153}$

\section{Multi-Responsive St-Mc-DDSs}

Compared to single stimuli, multiple stimuli, such as the combination of Ex and En stimuli, may have synergistic effects. Wang et $\mathrm{al}^{73}$ combined magnetic stimulus and NIR light irradiation with redox responsiveness into a multistage targeted nanocarrier to enhance the efficacy of cancer therapy. The core-shell-S-S-shell nanocarrier was composed of an $\mathrm{Fe}_{3} \mathrm{O}_{4}$ core, an inward polydopamine (PDA, a photosensitizer) shell connected to TPP, and an outward methoxy PEG (mPEG) shell linked to PDA via a disulfide bond. The magnetic $\mathrm{Fe}_{3} \mathrm{O}_{4}$ core increased nanocarrier location in tumor. Once entering the tumor cells, the outer mPEG shell is detached by redox reaction to disclose TPP for Mc targeting. Upon NIR light irradiation, PDA generated a photothermal effect, and the loaded DOX rapidly entered the Mc, resulting in cell apoptosis. Chen et al ${ }^{58}$ combined NIR light stimuli and enzyme responsiveness to deliver dual peptide (Mc-targeting)-decorated AuNSs and DOX to cure tumors. Zhang et $\mathrm{al}^{76}$ combined NIR light stimuli and redox responsiveness to coprepare a Mc-targeting polymeric micelle.

The combination of different En stimuli (such as redox or $\mathrm{pH}$ and enzyme stimuli) may result in the sequential release of drugs or polymers at precise times. ${ }^{154}$ Zhou et al ${ }^{51}$ constructed lipid polymer nanocarriers containing PTX, which is composed of an amphipathic copolymer containing TPP, poly (D,L-lactide-co-glycolide) (PLGA) and an amphipathic copolymer having redox-responsive property. The hydrophobic drug core (CCM) was decorated with hydrophilic shell (OHA and TPP) via a disulfide bond. The micelles were ingested by cancerous cells through CD44 recognition, entered the Mc and released CCM due to disulfide bond cleavage in response to high levels of GSH. The long-acting PEGylated nanocarriers accumulated in the tumor. PEG4000 detached via redox-triggered activation after uptake by cancer cells. The nanocarriers recovered to carry positive charge, and then enhanced anticancer efficacy was achieved through precise localization at the Mc.

\section{Relationship Characteristics of St- Mc-DDSs}

According to the spatial location relationships of different types of St- or Mc-DDSs, St-Mc-DDS are mainly classified as one of 5 types (Figure 1C): St material loaded with a Mc group or molecule-drug St-Mc-DDS; St material-Mc group or molecule loaded with drug St-Mc-DDS; St material-Mc material loaded with drug St-Mc-DDS; Mc group or molecule-drug St-Mc-DDS; or normal material-loaded with St-Mc-DDS.

\section{St Material Loaded with Mc Group or Molecule-Drug}

The drug molecules conjugated with Mc-targeting groups or molecules are further encapsulated in St materials to form different types (such as pH-, redox-, enzyme- or light-responsive) of St-Mc-DDSs. The Mc group or molecule-drug will be released in response to the tumor microenvironment or local Ex stimulus, which overcomes the nonspecific drug uptake by normal cells to a certain extent, thereby reducing toxicity. However, the linkage of the Mc group or molecule to the drug molecule may have a negative effect on anticancer efficacy. Therefore, an anticancer efficacy comparison between the Mc group or molecule-drug and free drug group is necessary and valuable.

Zhang et $\mathrm{al}^{42}$ synthesized a docetaxel-TPP (a Mc-targeting molecule) conjugate, and incorporated it into liposomes composed of PEG-Schiff base-cholesterol (a pHsensitive St material) and DSPE. PEG-Schiff base-cholesterol was hydrolyzed in $\mathrm{pH} \sim 6.0$ (tumor microenvironment) to get rid of PEG shell, and DSPE merged with the tumor lysosomal membrane $(\mathrm{pH} \sim 5.0)$, resulting in fast drug release into the cytoplasm and accumulation into the Mc under the guidance of TPP. Xing et $\mathrm{al}^{46}$ developed amphiphilic quercetin-TPP conjugates into a selfassembled nanoparticles composed of phenylboronic acid-PEG (a pH-sensitive St material) via boric acid ester bonds for tumor therapy. Zhang et $\mathrm{al}^{76}$ encapsulated PPA (a photosensitizer)-TPP (a Mc-targeting molecule) into imidazole (a redox-responsive St material)-bearing polymeric micelles. Song et $\mathrm{al}^{19}$ constructed a nanodrug selfassembled from a 9-O-octadecyl (a Mc-targeting group)substituted berberine derivative, further modified with DSPE-PEG2000 to increase stability and coated the nanodrug with HA (an enzyme HAase-sensitive St material) to achieve tumor targeting. Chen et al coencapsulated DOX and TPP-KLA (a Mc-targeting molecule and peptide)- 
decorated AuNSs (a light-responsive Sm material) into a HA (an enzyme HAase-sensitive St material) shell. ${ }^{58}$

\section{St Material-Mc Group or Molecules Loaded with Drug}

The Mc targeting group or molecule is directly bonded to a St material to form a composite material that will further encapsulate drug molecules to form different types (such as pH-, redox- or enzyme-responsive) of St-Mc-DDSs. Tan et $\mathrm{al}^{43}$ conjugated a lipophilic cation CTPP (a Mctargeting molecule) with CSOSA (an alkaline $\mathrm{pH}$-sensitive St material) to produce a St-Mc-material (CTPP-CSOSA) that formed micelles that simultaneously encapsulated celastrol into the hydrophobic core. Jiang et $\mathrm{al}^{47}$ conjugated the Mc peptide KLA with DSPE and DMA (a pHresponsive material) to yield a DSPE-KLA-DMA lipid to prepare liposomes containing PTX. Zhou et $\mathrm{al}^{51}$ mixed C18-PEG2000-TPP (a Mc group) and DLPE-SSmPEG4000 (a reductive responsive polymer) with PLGA to prepare nanoparticles loaded with PTX. Pramanik et $\mathrm{al}^{52}$ selected a TPP-modified nonisocyanate polyurethane (an esterase-responsive polymer) to construct biodegradable nanocapsules containing DOX.

As far as St-Mc-DDSs with St material-Mc group or molecules loaded with drugs are concerned, the antitumor effects of the drug can retain intact since it has an unmodified structure. However, Mc groups or molecules are usually positively charged, easily absorbed by non-specific proteins and rapidly eliminated by the reticuloendothelial system in circulation. ${ }^{47,51,57}$ Therefore, it is necessary to cover a Mc material with a negatively charged St material. In addition, when reaching the tumor site, the St material should be able to expose the Mc groups or molecules in response to stimuli, which can promote the electrostatic interaction between the nanoparticles and cancer cell membrane for cell internalization.

\section{St Material-Mc Material Loaded with Drug}

St material-Mc material can be a compound material composed of St- and Mc-material ${ }^{45}$ or a material that has both smart properties and Mc-targeting abilities. ${ }^{115,119}$

Shi et $\mathrm{al}^{36}$ incorporated the HER-2 peptide-PEG2000Schiff base-cholesterol (HPSC) derivative (a pH-responsive material) on the surface of DQAsomes (dicationic dequalinium vesicle, a Mc material) containing DOX to treat drug-resistant breast cancer. Li et $\mathrm{al}^{45}$ encapsulated docetaxel in a positively charged mesoporous silica nanoparticle core (MSN, a Mc material) and then wrapped it with a $\mathrm{pH}$-responsive HPMA copolymer shell to cover the positive charge of the mesoporous silica MSN. When StMc-DDS is prepared using a compound of St material-Mc material, ${ }^{36}$ its structural characteristics and in vivo processes are similar to those of St-Mc-DDSs containing a drug loaded in St material-Mc group or molecule. ${ }^{47,51} \mathrm{St}$ material-Mc material St-Mc-DDSs usually respond to stimulation of the tumor microenvironment or local Ex stimuli and induce physical property changes, such as particle size and charge. Then, the St-Mc-DDS arrives at the Mc under the guidance of the Mc material.

Carbon nanomaterials can be used not only as Mctargeted nanocarriers but also as $\mathrm{St}$ photosensitizers to induce phototherapy. ${ }^{119}$ Han et al $^{115}$ constructed a hypericin-functionalized NGO to deliver DOX, which had enhanced Mc targeting and synergistic anticancer effects. St-Mc-DDSs prepared using a single material having both Sm properties and a Mc targeting ability usually first accumulates in the Mc, and then energy conversion occurs under Ex stimulation. ${ }^{115,119}$

\section{Mc Group or Molecule-St Material-Drug}

When the Mc group or molecule, St material and drug are connected together, they form a St-Mc-DDS with a $100 \%$ encapsulation rate. Wang et $\mathrm{al}^{50}$ proposed TPP-OHA-S-SCCM micelles (an Mc molecule-enzyme/redox-sensitive multifunctional micelle) to exert anticancer efficacy. $\mathrm{Li}$ et $\mathrm{al}^{44}$ presented TPP-fluorogen-hydrazone bond-PEG micelles (an Mc molecule-pH sensitive micelle), and fluorogen underwent aggregation-induced emission (AIE). The PEG moiety increased blood circulation stability. Guan et $\mathrm{al}^{57}$ decorated AIE copolymers (PAIE, a photosensitizer able to be photoactivated upon $980 \mathrm{~nm}$ laser irradiation to yield ROS) with TPP to form PAIETPP, which was further conjugated with mPEG-CHO (a $\mathrm{pH}$-responsive material) via a benzoic imine bond. Wang et $\mathrm{al}^{73}$ prepared TPP-PDA (a photothermal agent)-S-S (redox sensitive)-mPEG nanoparticles using a similar strategy.

St-Mc-DDSs formed by directly connecting a drug with the Mc group or molecule-St material may reduce the problem of drug leakage during circulation. These compounds can not only change the physical properties in response to stimulation of the tumor microenvironment but also deliver therapeutic agents to produce $\mathrm{PDT}^{44,57}$ and $\mathrm{PTT}^{73}$ effects under light irradiation. 


\section{Normal Material Loaded with St-Mc-Drug} (St-Mc) drugs mainly refer to small molecule drugs with Mc targeting ability and light conversion performance. They can be encapsulated inside common materials to form St-Mc-DDSs. IR-780 is a St-Mc drug that can selectively accumulate in the $\mathrm{Mc}$ and is commonly used in photothermography, PTT, and PDT. ${ }^{54,56,86}$ Zhang et al ${ }^{54}$ encapsulated IR780 into nanoliposomes based on perfluorooctyl bromide to form an artificial nanoRBC. These liposomes delivered oxygen to the tumor to alleviate tumor hypoxia and enhanced Mc-targeted phototherapy and multiple imaging guidance/monitoring. The iridium (Ir) complex is another St-Mc drug that targets the Mc and can achieve photoredox reaction in tumor. ${ }^{55,155-157}$ Huang et $\mathrm{al}^{157}$ prepared an Ir photocatalyst. This complex localizes in the Mc and depletes NADH, unbalances intracellular redox and causes immunogenic cellular death upon light irradiation. In another work, $\mathrm{Ir}$ and $\mathrm{Fe}_{3} \mathrm{O}_{4}$ cooperated to form photothermogenic nanozyme $\mathrm{Ir} @ \mathrm{Fe}_{3} \mathrm{O}_{4} \quad \mathrm{NPs}$, which could increase the local temperature of the tumor, thereby catalyzing $\mathrm{H}_{2} \mathrm{O}_{2}$ to generate $\mathrm{OH} .{ }^{55}$ Similarly, PEGylated SWCNTs were used for Mc-targeted PTD. ${ }^{68}$

Compared with the above four types of St-Mc-DDSs, St-Mc-DDSs with St-Mc drug loading in common materials has a simpler design and preparation requirements for dosage forms. They can even be administered directly. ${ }^{157}$ In general, it is necessary to prepare St-Mc-DDSs with suitable structural properties based on the properties of the given drug and material.

\section{Clinical Research Progress}

PDT is considered a prospect effective therapy without obvious side effects. Photofrin ${ }^{\circledR}$ (a hematoporphyrinderived photosensitizer) is a powder injection to cure various cancers such as colorectal carcinoma, ${ }^{158}$ esophageal cancer, ${ }^{159}$ and malignant cutaneous neoplasms. ${ }^{160}$ The application of micelle nanotechnology further enhances Photofrin $^{\circledR}$ delivery and efficacy at the cellular level. ${ }^{161}$ Visudyne $^{\circledR}$ (a liposomal photosensitizer containing a second-generation photosensitizer derived from porphyrin) is clinically used to treat subfoveal choroidal neovascularization ${ }^{162,163}$ and has been found to be preclinically effective for cancer treatment. ${ }^{164}$ Ameluz ${ }^{\circledR}$ (containing $10 \%$ of the photosensitizer 5 -aminolevulinic acid) is a nonsterile white-to-yellow nanoemulsion-based gel for topical use to clinically detect and treat bladder cancer ${ }^{165,166}$ or cure melanoma skin cancer. ${ }^{167}$ In addition to light- responsive St-DDSs, other stimuli-responsive St-DDSs are developed in different clinical phases. They are thermoresponsive liposomes ThermoDox to cure breast tumor (phase II) and liver cancer (phase III); the enzyme-responsive polymeric nanoparticles Opaxio to cure ovarian carcinoma; and the magnetic field-responsive iron oxide NanoTherm for the treatment of glioblastoma. One significant characteristic of these promising St-DDSs is their simple formulation, which favors their preclinical to clinical transformation. ${ }^{168,169}$

Some synthesized Mc-targeted compounds, such as MitoQ and SkQ1 (also called Visomitin), have already entered the clinical trial stage. Both are lipophilic antioxidants with TPP groups. ${ }^{170}$ MitoQ is used to treat aging, ${ }^{171}$ pulmonary hypertension, etc., ${ }^{172}$ while SkQ1 is used to treat conjunctivitis ${ }^{173}$ in completed clinical trials. MitoQ is currently undergoing other clinical trials for pulmonary hypertension, etc. Unfortunately, all antioxidant tests are not for antitumor therapy. However, it is worth trying to perform the clinical transformation of other Mc-targeted compounds with TPP groups and their DDSs. ${ }^{34}$ Some novel compounds with potent anticancer activity have been identified to use the Mc as a target and act on its metabolism in recent years. Venetoclax represents a firstin-class selective and effective Bcl-2 inhibitor. ${ }^{174}$ It was approved by the United States FDA in 2016 to treat relapsed-refractory chronic lymphocytic leukemia. Its clinical dosage form is ordinary tablets. Ganetespib is an injectable small molecule drug. It has a favorable safety profile and promising early results by inhibiting heat shock protein 90. It has been investigated in multiple clinical trials of various tumors, such as metastatic pancreatic cancer (phase II), ${ }^{175}$ relapsed-refractory small cell lung cancer (phase $\mathrm{Ib} / \mathrm{II}),{ }^{176}$ and advanced carcinomas and sarcomas (phase I). ${ }^{177}$

To date, there are still no clinical antitumor investigations of St-Mc-DDSs or Mc-DDSs. In addition, there are only a few St-DDSs in the clinic or the clinical trial stage. Most of these compounds are still at the basic research or preclinical stages. The possible reasons are listed as follows.

1. Insufficient in vitro pharmaceutical data. The formulation consists of some excipients or critical materials that are not pharmaceutical grade and not approved for pharmaceutical application, and their components, purity, quality, function and toxicity are unclear. Most preparation processes are too 
sophisticated for industrial production scale-up. The drug payload and encapsulation efficiency of most St-Mc-DDSs have not been determined, and some may not be high. Formulation stability has not been considered and investigated.

2. Insufficient in vitro release, in vivo pharmacokinetics and biodistribution data. Some in vitro release in suitable release media should be supplemented, and the media type should be chosen based on administrative pathways and designed release microenvironment. There is no in vivo pharmacokinetic behavior research or analysis of the bioavailability or pharmacokinetic parameters, such as the area under the plasma drug concentration versus time curve, peak time, clearance, etc. The relationship between the in vivo pharmacokinetics and in vitro release should be investigated. The biodistribution in different normal tissues and tumors, tissue fluids, cells, and organelles, particularly the Mc, should be clarified.

3. Insufficient pharmacodynamic data. Most studies are limited to one or two cell lines and/or several animal experiments and are still in the proof-of-principle stage. The effects of stimuli and Mc targeting on intracellular trafficking, action pathways, and clinical efficacy require further research. When targeting the Mc alone does not achieve the desired therapeutic effect, designing nanocarriers that target multiple organelles at the same time may be an alternative strategy since in-depth studies on lysosomes, the endoplasmic reticulum and other organelles have shown that these organelles are closely related to apoptosis or autophagy, which can affect tumorigenesis.

4. Insufficient safety data. No preliminary safety evaluation of the Sm-Mc-DDS including toxicity, immunogenicity and side effects in animals has been reported. Additionally, the positive stimuli to rats or mice may not be suitable or safe for patients.

5. The mutual interference or promotion of St stimuli and Mc-targeting in one DDS requires more experimental data. Theoretically, each Sm type may combine with each Mc targeting type or subtype, but in fact, there have been no reports of some En (other biomolecules outside our list) or Ex (temperature, ultrasound, electric pulse, etc.) stimuli-responsive St-Mc-DDSs. The chosen criterion is not clear.

6. Compared to single stimulus-response St-Mc-DDSs, multiple stimuli-response St-Mc-DDSs are more difficult to achieve transformation. The latter is still in its infancy, their design principles are more complex than the former, and their fabrication and assembly processes are more difficult. Sometimes, failure to respond to one stimulus may lead to ineffectiveness of the whole system. Therefore, the sequence and degree of response to each stimulus should be further assessed.

\section{Summary and Outlook}

Mc dysfunction plays a vital role in programmed cell death, such as apoptosis and necrosis. It is reasonable to choose the Mc as a novel target for an antitumor strategy. However, most drugs or nanocarriers on the market do not have Mc targeting functions, and they need to overcome many obstacles before reaching the tumor tissue, cells and Mc due to the complexity of the tumor tissue environment. The concept of Mc targeting, while seemingly simple in theory, has multiple subtly different practical approaches. Lipophilic cation such as TPP and IR-780 have good safety and clinical potential. IR-780 are also used for PDT/PTT therapy. Mc-targeting peptides such as KLA and MQ peptides, have positively charged and lipophilic amino acids. Mc-targeting aptamers are too expensive to make clinical transformation difficult. Peptides and aptamers may be unstable and cause unwanted side-effects due to large molecular weights. Mc materials form vesicles to deliver drug. NGO nanoparticles may achieve PDT/PTT effects. There are differences on the future status of various Mc-targeted approaches to cancer therapy. In this context, structurally modified and programmed micro/ nanoparticles, which can be programmed using computation techniques to obtain En ( $\mathrm{pH}$, redox and enzyme) or Ex (light and magnetism) stimuli responsive to increase their accumulation in the Mc. Multifunctional nanocarriers with single/multiple stimuli-responsiveness and Mc targeting properties may better protect and deliver drugs, reduce normal tissue accumulation and enhance therapeutic effects. Stimuli-responsive system may be an effective way to improve Mc targeting delivery. An important biological hypothesis is that the tissue microenvironment can trigger a desirable event to a large extent from stimuliresponsive behavior. However, until now, no evidence supports this. The responsiveness should be considered as an important contributor to therapeutic efficacy, and an urgent necessity to assess in depth in vivo responsiveness that is intimately relevant for the functionality. Some 
strategies had been proposed to amplify the responsiveness and improve the functionality. ${ }^{178-180}$

Undoubtedly, enormous efforts have been exerted in the design and basic research of St-Mc-DDS for cancer therapy, and the preliminary results are encouraging. However, the clinical transformation of St-Mc-DDSs is comparatively motionless. In order to address the challenges in clinical transformation, there is a great need to consider and perform the following measures in the future, which include but are not limited to choosing the drug using the Mc as a main target; choosing approved pharmaceutical excipients to formulate the St-Mc-DDS; choosing suitable stimuli according to the tumor site (superficial or deep tumor); optimizing the preparation method and choosing a method that is as simple as possible; and performing the necessary experiments (such as stability, pharmacokinetics and safety tests) needed for clinical trial application. Recently, advances in machine learning and artificial intelligence immensely decode and empower the cell-nanomaterial interaction modelling, which give modern to nanomedicine to predict the targeting and efficacy of payload to intracellular compartment ${ }^{181-183}$ using in-silico methods. ${ }^{61}$ This potentially decipher the quantitative nanostructure activity-relationship (Nano-QSAR) and promote the understanding of bio-physicochemical identity at the nano-bio interface. In this context, structurally modified and programmed micro/nanoparticles, which can be programmed using computation techniques to stimuli responsive and increase their accumulation in the mitochondria. The predictability of targeting and effectiveness, coupled with the clarity of the mechanism of action, may accelerate the clinical transformation of nanomedicine. We should follow innovative advantages and conduct prospective research. In addition, considering the fact that more than $10 \%$ of all cancer drugs in use today are nanodrug and irony is less $10 \%$ of clinical oncologist know this, future concept must evolve around the why there is a need for education and training in nanomedicine for future doctors? Emphasis must be their incorporation into the general medical curriculum the key concept in nanomedicine.

Role of EPR in cancer barrier is somewhat oversell considering less than $1 \%$ nanomedicine formulations follow the trend designed by using EPR. In the last decades, it has been increasingly recognized that there is large interand intra-individual heterogeneity in EPR-mediated tumor targeting, explaining the heterogeneous outcomes of clinical trials in which nanomedicine formulations have been evaluated. To address this heterogeneity, as in other areas of oncology drug development, we have to move away from a one-size-fits-all tumor targeting approach, towards methods that can be employed to individualize and improve nanomedicine treatments.

Overall, St-Mc-DDSs may be innovative and sensitive precision medicines. They provide great potential for enhanced cancer treatment. This new strategy is expected to be applied in clinical practice as soon as possible and to open up new ideas for precision medicine soon.

\section{Abbreviations}

AIE, aggregation-induced emission; APases, alkaline phosphatases; AuNSs, gold nanostars; BD, berberine derivative; CCM, curcumin; CPPs, cell penetrating peptides; CTPP, (4-carboxybutyl) triphenylphosphonium bromide; Cyt, cytochrome; DDSs, drug delivery systems; DMA, dimethylmaleic anhydride; DOPE, dioleoyl phosphoethanolamine; DOX, doxorubicin; DSPE,1,2distearoyl-sn-glycero-3-phosphoethanolamine; EGFR, epidermal growth factor receptor; En, endogenous; En/ Sm-Mc-DDS, En stimuli-responsive $\mathrm{Sm}$ and Mc-targeted platforms; EphA 10, Eph receptor A10; EPR, enhanced permeability and retention effect; Ex, exogenous; GSH, glutathione; HAases, hyaluronidases; HPMA, N-(2-hydroxypropyl) methacrylamide; HPSC, HER-2 peptide-PEG2000-Schiff base-cholesterol; Hsp90, heat shock protein 90; ICG, indocyanine green; LPNPs, lipid polymer hybrid nanoparticles; mAb, monoclonal antibody; Mc, mitochondria; MDR, multidrug resistance; mPEG, methoxy PEG; MPP, Mc-penetrating peptide; MSN, mesoporous silica nanoparticle; MTS, Mctargeting sequence; NGO, graphene oxide; NIPU, nonisocyanate polyurethane nanocapsule; NIR, near-infrared; OHA, oligomeric hyaluronic acid; PDA, polydopamine; PDT, photodynamic therapy; PEG, polyethylene glycol; PFOB, perfluorooctyl bromide; PLGA, poly (D,L-lactide-co-glycolide); PPA, pyropheophorbide A; PTT, photothermal therapy; PTX, paclitaxel; ROS, reactive oxygen species; St, stimuli-responsive; StDDSs, stimuli-responsive drug delivery systems; StMc-DDSs, smart stimuli-responsive and mitochondriatargeting drug delivery systems; SPIONs, superparamagnetic iron oxide nanoparticles; SS, Szeto-Schiller; SWCNs, single-walled carbon nanotubes; TAT, transactivating transcriptional activator; TPP, triphenylphosphonium; UCNP, upconversion nanoparticle; UV, ultraviolet. 


\section{Acknowledgments}

This research is partially supported by grants from National Natural Science Foundation of China (30973645), Chongqing Science and Technology Committee (cstc2017shmsA130028, cstc2015jcyjBX0037) and Chongqing Education Committee (CYS20212, CYS19210).

\section{Disclosure}

The authors declare no conflict of interest.

\section{References}

1. Siegel RL, Miller KD, Jemal A. Cancer statistics, 2019. $C A$ Cancer J Clin. 2019;69:7-34. doi:10.3322/caac.21551

2. Zhao J, Li Y, He D, et al. Natural oral anticancer medication in small ethanol nanosomes coated with a natural alkaline polysaccharide. ACS Appl Mater Interfaces. 2020;12:16159-16167. doi:10.1021/acsami.0c02788

3. Zhang Y, Yu J, Bomba HN, Zhu Y, Gu Z. Mechanical forcetriggered drug delivery. Chem Rev. 2016;116:12536-12563. doi:10.1021/acs.chemrev.6b00369

4. Huang Y, Gu J, Yan Z, et al. Cytomembrane-mimicking nanocarriers with a scaffold consisting of a cd44-targeted endogenous component for effective asparaginase supramolecule delivery. Nanoscale. 2020;12:12083-12097. doi:10.1039/ D0NR02588G

5. Biswas S, Torchilin VP. Nanopreparations for organelle-specific delivery in cancer. Adv Drug Deliv Rev. 2014;66:26-41. doi:10.1016/j.addr.2013.11.004

6. Li J, Kataoka K. Chemo-physical strategies to advance the in vivo functionality of targeted nanomedicine: the next generation. $J \mathrm{Am}$ Chem Soc. 2021;143:538-559. doi:10.1021/jacs.0c09029

7. Chen B, Dai W, He B, et al. Current multistage drug delivery systems based on the tumor microenvironment. Theranostics. 2017;7:538-558. doi:10.7150/thno.16684

8. Deng Y, Ling J, Li MH. Physical stimuli-responsive liposomes and polymersomes as drug delivery vehicles based on phase transitions in the membrane. Nanoscale. 2018;10:6781-6800. doi:10.1039/C8NR00923F

9. Zhang $\mathrm{X}$, Chen $\mathrm{L}$, Lim KH, et al. The pathway to intelligence: using stimuli-responsive materials as building blocks for constructing smart and functional systems. Adv Mater. 2019;31: e1804540. doi:10.1002/adma.201804540

10. Wu J, Li J, Wang H, Liu CB. Mitochondrial-targeted penetrating peptide delivery for cancer therapy. Expert Opin Drug Deliv. 2018;15:951-964. doi:10.1080/17425247.2018.1517750

11. Zong WX, Rabinowitz JD, White E. Mitochondria and cancer Mol Cell. 2016;61:667-676. doi:10.1016/j.molcel.2016.02.011

12. Wallace DC. Mitochondria and cancer. Nat Rev Cancer. 2012;12:685-698. doi:10.1038/nrc3365

13. Liu PS, Ho PC. Mitochondria: a master regulator in macrophage and t cell immunity. Mitochondrion. 2018;41:45-50. doi:10.1016/ j.mito.2017.11.002

14. Vyas S, Zaganjor E, Haigis MC. Mitochondria and cancer. Cell. 2016;166:555-566. doi:10.1016/j.cell.2016.07.002

15. Gururaja Rao S. Mitochondrial changes in cancer. Handb Exp Pharmacol. 2017;240:211-227.

16. Liu G, Zhao X, Zhang Y, et al. Engineering biomimetic platesomes for ph-responsive drug delivery and enhanced antitumor activity. Adv Mater. 2019;31:e1900795. doi:10.1002/adma.20 1900795
17. Liu X, Wang L, Xu X, Zhao H, Jiang W. Endogenous stimuliresponsive nucleus-targeted nanocarrier for intracellular mrna imaging and drug delivery. ACS Appl Mater Interfaces. 2018;10:39524-39531. doi:10.1021/acsami.8b16345

18. Kienzle A, Kurch S, Schloder J, et al. Dendritic mesoporous silica nanoparticles for ph-stimuli-responsive drug delivery of tnf-alpha. Adv Healthc Mater. 2017;6. doi:10.1002/adhm.201700012

19. Song J, Lin C, Yang X, et al. Mitochondrial targeting nanodrugs self-assembled from 9-o-octadecyl substituted berberine derivative for cancer treatment by inducing mitochondrial apoptosis pathways. J Control Release. 2019;294:27-42. doi:10.1016/j. jconrel.2018.11.014

20. Yang G, Xu L, Xu J, et al. Smart nanoreactors for ph-responsive tumor homing, mitochondria-targeting, and enhanced photodynamic-immunotherapy of cancer. Nano Lett. 2018;18:24752484. doi:10.1021/acs.nanolett.8b00040

21. Arrieta O, Barron F, Padilla MS, et al. Effect of metformin plus tyrosine kinase inhibitors compared with tyrosine kinase inhibitors alone in patients with epidermal growth factor receptormutated lung adenocarcinoma: a Phase 2 randomized clinical trial. JAMA Oncol. 2019;5:e192553. doi:10.1001/jamaoncol.20 19.2553

22. Charbgoo F, Nikkhah M, Behmanesh M. Size of single-wall carbon nanotube affects the folate receptor-mediated cancer cell targeting. Biotechnol Appl Biochem. 2018;65:328-337. doi:10. 1002/bab.1592

23. Xu L, Bai Q, Zhang X, Yang H. Folate-mediated chemotherapy and diagnostics: an updated review and outlook. $J$ Control Release. 2017;252:73-82. doi:10.1016/j.jconrel.2017.02.023

24. Choudhury H, Pandey M, Chin PX, et al. Transferrin receptorstargeting nanocarriers for efficient targeted delivery and transcytosis of drugs into the brain tumors: a review of recent advancements and emerging trends. Drug Deliv Transl Res. 2018;8:15451563. doi:10.1007/s13346-018-0552-2

25. Tortorella S, Karagiannis TC. Transferrin receptor-mediated endocytosis: a useful target for cancer therapy. J Membr Biol. 2014;247:291-307. doi:10.1007/s00232-014-9637-0

26. Guo X, Wang L, Duval K, Fan J, Zhou S, Chen Z. Dimeric drug polymeric micelles with acid-active tumor targeting and frettraceable drug release. Adv Mater. 2018;30.

27. Zhang Y, Li H, Wang Q, et al. Rationally designed selfassembling nanoparticles to overcome mucus and epithelium transport barriers for oral vaccines against helicobacter pylori. Adv Funct Mater. 2018;28:1802675. doi:10.1002/adfm.2018 02675

28. Fields RJ, Quijano E, McNeer NA, et al. Modified poly(lactic-coglycolic acid) nanoparticles for enhanced cellular uptake and gene editing in the lung. Adv Healthc Mater. 2015;4:361-366. doi:10.1002/adhm.201400355

29. Almarwani B, Phambu EN, Alexander C, Nguyen HAT, Phambu $\mathrm{N}$, Sunda-Meya A. Vesicles mimicking normal and cancer cell membranes exhibit differential responses to the cell-penetrating peptide pep-1. Biochim Biophys Acta Biomembr. 2018;18 60:1394-1402. doi:10.1016/j.bbamem.2018.03.022

30. Pescina S, Sala M, Padula C, et al. Design and synthesis of new cell penetrating peptides: diffusion and distribution inside the cornea. Mol Pharm. 2016;13:3876-3883. doi:10.1021/acs. molpharmaceut.6b00658

31. Rhee M, Davis P. Mechanism of uptake of c105y, a novel cellpenetrating peptide. $J$ Biol Chem. 2006;281:1233-1240. doi:10.1074/jbc.M509813200

32. Xi J, Li M, Jing B, et al. Long-circulating amphiphilic doxorubicin for tumor mitochondria-specific targeting. ACS Appl Mater Interfaces. 2018;10:43482-43492. doi:10.1021/acsami. 8 b17399 
33. Lu P, Bruno BJ, Rabenau M, Lim CS. Delivery of drugs and macromolecules to the mitochondria for cancer therapy. J Control Release. 2016;240:38-51. doi:10.1016/j.jconrel.2015.10.023

34. Zielonka J, Joseph J, Sikora A, et al. Mitochondria-targeted triphenylphosphonium-based compounds: syntheses, mechanisms of action, and therapeutic and diagnostic applications. Chem Rev. 2017;117:10043-10120. doi:10.1021/acs.chemrev.7b 00042

35. Ishikawa T, Somiya K, Munechika R, Harashima H, Yamada Y. Mitochondrial transgene expression via an artificial mitochondrial DNA vector in cells from a patient with a mitochondrial disease. $J$ Control Release. 2018;274:109-117. doi:10.1016/j.jconrel.2018. 02.005

36. Shi M, Zhang J, Li X, et al. Mitochondria-targeted delivery of doxorubicin to enhance antitumor activity with her-2 peptide-mediated multifunctional ph-sensitive dqasomes. Int $J$ Nanomedicine. 2018;13:4209-4226. doi:10.2147/IJN.S1 63858

37. Chuah J-A, Matsugami A, Hayashi F, Numata K. Self-assembled peptide-based system for mitochondrial-targeted gene delivery: functional and structural insights. Biomacromolecules. 2016;17:3547-3557. doi:10.1021/acs.biomac.6b01056

38. Yamada Y, Harashima H. Mitochondrial drug delivery systems for macromolecule and their therapeutic application to mitochondrial diseases. Adv Drug Deliv Rev. 2008;60:1439-1462. doi:10.1016/j.addr.2008.04.016

39. Nam HY, Hong JA, Choi J, et al. Mitochondria-targeting peptoids. Bioconjug Chem. 2018;29:1669-1676. doi:10.1021/acs. bioconjchem. 8 b00148

40. Laquintana V, Denora N, Lopalco A, et al. Translocator protein ligand-plga conjugated nanoparticles for 5-fluorouracil delivery to glioma cancer cells. Mol Pharm. 2014;11:859-871. doi:10.1021/ mp400536z

41. Iacobazzi RM, Lopalco A, Cutrignelli A, et al. Bridging pharmaceutical chemistry with drug and nanoparticle targeting to investigate the role of the 18-kda translocator protein tspo. ChemMedChem. 2017;12:1261-1274. doi:10.1002/cmdc.201700322

42. Zhang J, Yang C, Pan S, et al. Eph a10-modified ph-sensitive liposomes loaded with novel triphenylphosphine-docetaxel conjugate possess hierarchical targetability and sufficient antitumor effect both in vitro and in vivo. Drug Deliv. 2018;25:723-737. doi:10.1080/10717544.2018.1446475

43. Tan Y, Zhu Y, Zhao Y, et al. Mitochondrial alkaline ph-responsive drug release mediated by celastrol loaded glycolipid-like micelles for cancer therapy. Biomaterials. 2018;154:169-181. doi:10.1016/ j.biomaterials.2017.07.036

44. Li J, Liu Y, Li H, et al. Ph-sensitive micelles with mitochondriatargeted and aggregation-induced emission characterization: synthesis, cytotoxicity and biological applications. Biomater Sci. 2018;6:2998-3008. doi:10.1039/C8BM00889B

45. Li L, Sun W, Li L, et al. A ph-responsive sequential-disassembly nanohybrid for mitochondrial targeting. Nanoscale. 2017;9:314325. doi:10.1039/C6NR07004C

46. Xing L, Lyu JY, Yang Y, et al. Ph-responsive de-pegylated nanoparticles based on triphenylphosphine-quercetin self-assemblies for mitochondria-targeted cancer therapy. Chem Commun (Camb). 2017;53:8790-8793. doi:10.1039/C7CC04058J

47. Jiang L, Li L, He X, et al. Overcoming drug-resistant lung cancer by paclitaxel loaded dual-functional liposomes with mitochondria targeting and ph-response. Biomaterials. 2015;52:126-139. doi:10.1016/j.biomaterials.2015.02.004

48. Yu P, Yu H, Guo C, et al. Reversal of doxorubicin resistance in breast cancer by mitochondria-targeted ph-responsive micelles. Acta Biomater. 2015;14:115-124. doi:10.1016/j.actbio.2014. 12.001
49. Mo R, Sun Q, Xue J, et al. Multistage ph-responsive liposomes for mitochondrial-targeted anticancer drug delivery. Adv Mater. 2012;24:3659-3665. doi:10.1002/adma.201201498

50. Wang K, Qi M, Guo C, et al. Novel dual mitochondrial and cd44 receptor targeting nanoparticles for redox stimuli-triggered release. Nanoscale Res Lett. 2018;13:32. doi:10.1186/s11671018-2445-1

51. Zhou W, Yu H, Zhang LJ, et al. Redox-triggered activation of nanocarriers for mitochondria-targeting cancer chemotherapy. Nanoscale. 2017;9:17044-17053. doi:10.1039/C7NR06130G

52. Pramanik SK, Sreedharan S, Singh H, et al. Mitochondria targeting non-isocyanate-based polyurethane nanocapsules for enzymetriggered drug release. Bioconjug Chem. 2018;29:3532-3543. doi:10.1021/acs.bioconjchem.8b00460

53. Wang H, Feng Z, Wang Y, Zhou R, Yang Z, Xu B. Integrating enzymatic self-assembly and mitochondria targeting for selectively killing cancer cells without acquired drug resistance. J Am Chem Soc. 2016;138:16046-16055. doi:10.1021/jacs.6b09783

54. Zhang L, Wang D, Yang K, et al. Mitochondria-targeted artificial "nano-rbcs" for amplified synergistic cancer phototherapy by a single nir irradiation. Adv Sci (Weinh). 2018;5:1800049. doi:10.1002/advs.201800049

55. Qiu K, Wang J, Rees TW, Ji L, Zhang Q, Chao H. A mitochondria-targeting photothermogenic nanozyme for MRI-guided mild photothermal therapy. Chem Commun (Camb). 2018;54:1410814111. doi:10.1039/C8CC08570F

56. Yue C, Yang Y, Song J, et al. Mitochondria-targeting near-infrared light-triggered thermosensitive liposomes for localized photothermal and photodynamic ablation of tumors combined with chemotherapy. Nanoscale. 2017;9:11103-11118. doi:10.1039/C7NR02193C

57. Guan Y, Lu H, Li W, et al. Near-infrared triggered upconversion polymeric nanoparticles based on aggregation-induced emission and mitochondria targeting for photodynamic cancer therapy. ACS Appl Mater Interfaces. 2017;9:26731-26739. doi:10.1021/ acsami.7b07768

58. Chen S, Lei Q, Qiu WX, et al. Mitochondria-targeting "nanoheater" for enhanced photothermal/chemo-therapy. Biomaterials. 2017;117:92-104. doi:10.1016/j.biomaterials.2016.11.056

59. Song XF, Tian H, Zhang P, Zhang ZX. Expression of cyt-cmediated mitochondrial apoptosis-related proteins in rat renal proximal tubules during development. Nephron. 2017;135:7786. doi:10.1159/000450585

60. Chakrabortty S, Sison M, Wu Y, et al. Nir-emitting and photothermal active nanogold as mitochondria-specific probes. Biomater Sci. 2017;5:966-971. doi:10.1039/C6BM00951D

61. Singh AV, Jahnke T, Wang S, et al. Anisotropic gold nanostructures: optimization via in silico modeling for hyperthermia. ACS Appl Nano Mater. 2018;1:6205-6216. doi:10.1021/acsanm.8b01406

62. Singh AV, Alapan Y, Jahnke T, et al. Seed-mediated synthesis of plasmonic gold nanoribbons using cancer cells for hyperthermia applications. J Mater Chem B. 2018;6:7573-7581. doi:10.1039/ C8TB02239A

63. Singh AV, Batuwangala M, Mundra R, et al. Biomineralized anisotropic gold microplate-macrophage interactions reveal frustrated phagocytosis-like phenomenon: a novel paclitaxel drug delivery vehicle. ACS Appl Mater Interfaces. 2014;6:1467914689. doi:10.1021/am504051b

64. Wei Y, Zhou F, Zhang D, Chen Q, Xing D. A graphene oxide based smart drug delivery system for tumor mitochondria-targeting photodynamic therapy. Nanoscale. 2016;8:3530-3538. doi:10.1039/C5NR07785K

65. Hou Z, Zhang Y, Deng K, et al. UV-emitting upconversion-based tio2 photosensitizing nanoplatform: near-infrared light mediated in vivo photodynamic therapy via mitochondria-involved apoptosis pathway. ACS Nano. 2015;9:2584-2599. doi:10.1021/nn506107c 
66. Jung HS, Han J, Lee JH, et al. Enhanced nir radiation-triggered hyperthermia by mitochondrial targeting. $\mathrm{J}$ Am Chem Soc. 2015;137:3017-3023. doi:10.1021/ja5122809

67. Ju E, Li Z, Liu Z, Ren J, Qu X. Near-infrared light-triggered drug-delivery vehicle for mitochondria-targeted chemo-photothermal therapy. ACS Appl Mater Interfaces. 2014;6:4364-4370. doi:10.1021/am5000883

68. Zhou F, Wu S, Wu B, Chen WR, Xing D. Mitochondria-targeting single-walled carbon nanotubes for cancer photothermal therapy. Small. 2011;7:2727-2735. doi:10.1002/smll.201100669

69. Kim YK, Zhang M, Lu JJ, et al. Pk11195-chitosan-graft-polyethylenimine-modified spion as a mitochondria-targeting gene carrier. J Drug Target. 2016;24:457-467. doi:10.3109/10611 86X.2015.1087527

70. Zhang Y, Shen Y, Teng X, Yan M, Bi H, Morais PC. Mitochondria-targeting nanoplatform with fluorescent carbon dots for long time imaging and magnetic field-enhanced cellular uptake. ACS Appl Mater Interfaces. 2015;7:10201-10212. doi:10.1021/acsami.5b00405

71. Choi J, Shin J, Lee J, Cha M. Magnetic response of mitochondriatargeted cancer cells with bacterial magnetic nanoparticles. Chem Commun (Camb). 2012;48:7474-7476. doi:10.1039/c2cc33659f

72. Oh Y, Je JY, Moorthy MS, Seo H, Cho WH. Ph and nir-lightresponsive magnetic iron oxide nanoparticles for mitochondriamediated apoptotic cell death induced by chemo-photothermal therapy. Int $J$ Pharm. 2017;531:1-13. doi:10.1016/j.ijpharm.20 17.07.014

73. Wang Y, Wei G, Zhang X, et al. Multistage targeting strategy using magnetic composite nanoparticles for synergism of photothermal therapy and chemotherapy. Small. 2018;14:e1702994. doi:10.1002/smll.201702994

74. Guo R, Peng H, Tian Y, Shen S, Yang W. Mitochondria-targeting magnetic composite nanoparticles for enhanced phototherapy of cancer. Small. 2016;12:4541-4552. doi:10.1002/smll.201601094

75. He H, Cattran AW, Nguyen T, Nieminen AL, Xu P. Tripleresponsive expansile nanogel for tumor and mitochondria targeted photosensitizer delivery. Biomaterials. 2014;35:9546-9553. doi:10.1016/j.biomaterials.2014.08.004

76. Zhang X, Yan Q, Mulatihan DN, et al. Pharmaceutical micelles featured with singlet oxygen-responsive cargo release and mitochondrial targeting for enhanced photodynamic therapy. Nanotechnology. 2018;29:255101. doi:10.1088/1361-6528/aabbdb

77. Singh AV, Hosseinidoust Z, Park BW, Yasa O, Sitti M. Microemulsion-based soft bacteria-driven microswimmers for active cargo delivery. ACS Nano. 2017;11:9759-9769. doi:10. 1021/acsnano.7b02082

78. Ma $\mathrm{P}$, Chen $\mathrm{J}, \mathrm{Bi} \mathrm{X}$, et al. Overcoming multidrug resistance through the glut1-mediated and enzyme-triggered mitochondrial targeting conjugate with redox-sensitive paclitaxel release. ACS Appl Mater Interfaces. 2018;10:12351-12363. doi:10.1021/ acsami.7b18437

79. Singh AV, Dad Ansari MH, Dayan CB, et al. Multifunctional magnetic hairbot for untethered osteogenesis, ultrasound contrast imaging and drug delivery. Biomaterials. 2019;219:119394. doi:10.1016/j.biomaterials.2019.119394

80. Singh AV. Commentary on "peptide-conjugated nanoparticles as targeted anti-angiogenesis therapeutic and diagnostic in cancer" by shaker a. Mousa, Pharmaceutical Research Institute, Albany College of Pharmacy and Health Sciences, Rensselaer, NY 12144, United States - peptide-conjugated nanoparticles for multimodal nanomedicine. Curr Med Chem. 2020;27:2927-2928. doi:10.21 74/092986732717200604120627

81. Chen K, Cai H, Zhang H, et al. Stimuli-responsive polymerdoxorubicin conjugate: antitumor mechanism and potential as nano-prodrug. Acta Biomater. 2019;84:339-355. doi:10.1016/j. actbio.2018.11.050
82. Guzman-Villanueva D, Weissig V. Mitochondria-targeted agents: mitochondriotropics, mitochondriotoxics, and mitocans. Handb Exp Pharmacol. 2017;240:423-438.

83. Qu Q, Ma X, Zhao Y. Targeted delivery of doxorubicin to mitochondria using mesoporous silica nanoparticle nanocarriers. Nanoscale. 2015;7:16677-16686. doi:10.1039/C5NR05139H

84. Murphy MP, Smith RA. Targeting antioxidants to mitochondria by conjugation to lipophilic cations. Annu Rev Pharmacol Toxicol. 2007;47:629-656. doi:10.1146/annurev.pharmtox.47.120 505.105110

85. Ma Z, Han K, Dai X, Han H. Precisely striking tumors without adjacent normal tissue damage via mitochondria-templated accumulation. ACS Nano. 2018;12:6252-6262. doi:10.1021/ acsnano.8b03212

86. Tan X, Luo S, Long L, et al. Structure-guided design and synthesis of a mitochondria-targeting near-infrared fluorophore with multimodal therapeutic activities. Adv Mater. 2017;29. doi:10. 1002/adma.201704196

87. Li K, Hou JT, Yang J, Yu XQ. A tumor-specific and mitochondria-targeted fluorescent probe for real-time sensing of hypochlorite in living cells. Chem Commun (Camb). 2017;53:5539-5541. doi:10.1039/C7CC01679D

88. Morimoto N, Takei R, Wakamura M, et al. Fast and effective mitochondrial delivery of omega-rhodamine-b-polysulfobetainepeg copolymers. Sci Rep. 2018;8:1128. doi:10.1038/s41598-01819598-2

89. Testai L, Barrese V, Soldovieri MV, et al. Expression and function of kv7.4 channels in rat cardiac mitochondria: possible targets for cardioprotection. Cardiovasc Res. 2016;110:40-50. doi:10.1093/ cvr/cvv281

90. Abunimer AN, Mohammed H, Cook KL, Soto-Pantoja DR, Campos MM, Abu-Asab MS. Mitochondrial autophagosomes as a mechanism of drug resistance in breast carcinoma. Ultrastruct Pathol. 2018;42:170-180. doi:10.1080/01913123.2017.1419328

91. Mordente A, Meucci E, Silvestrini A, Martorana GE, Giardina B. Anthracyclines and mitochondria. Adv Exp Med Biol. 2012;942:385-419.

92. Kavazis AN, Morton AB, Hall SE, Smuder AJ. Effects of doxorubicin on cardiac muscle subsarcolemmal and intermyofibrillar mitochondria. Mitochondrion. 2017;34:9-19. doi:10.1016/j. mito.2016.10.008

93. Agemy L, Friedmann-Morvinski D, Kotamraju VR, et al. Targeted nanoparticle enhanced proapoptotic peptide as potential therapy for glioblastoma. Proc Natl Acad Sci $U$ S A. 2011;108:17450-17455.

94. Kang YC, Son M, Kang S, et al. Cell-penetrating artificial mitochondria-targeting peptide-conjugated metallothionein 1a alleviates mitochondrial damage in parkinson's disease models. Exp Mol Med. 2018;50:105. doi:10.1038/s12276-018-0124-z

95. Woldetsadik AD, Vogel MC, Rabeh WM, Magzoub M. Hexokinase ii-derived cell-penetrating peptide targets mitochondria and triggers apoptosis in cancer cells. FASEB J. 2017;31:2168-2184. doi:10.1096/fj.201601173R

96. Alta RY, Vitorino HA, Goswami D, et al. Mitochondria-penetrating peptides conjugated to desferrioxamine as chelators for mitochondrial labile iron. PLoS One. 2017;12:e0171729. doi:10.1371/ journal.pone.0171729

97. Selmin F, Magri G, Gennari CG, Marchiano S, Ferri N, Pellegrino S. Development of poly(lactide-co-glycolide) nanoparticles functionalized with a mitochondria penetrating peptide. J Pept Sci. 2017;23:182-188. doi:10.1002/psc.2952

98. Jean SR, Ahmed M, Lei EK, Wisnovsky SP, Kelley SO. Peptidemediated delivery of chemical probes and therapeutics to mitochondria. Acc Chem Res. 2016;49:1893-1902. doi:10.1021/acs. accounts.6b00277 
99. Cerrato CP, Pirisinu M, Vlachos EN, Langel U. Novel cell-penetrating peptide targeting mitochondria. FASEB J. 2015;29:45894599. doi:10.1096/fj.14-269225

100. Toyama S, Shimoyama N, Szeto HH, Schiller PW, Shimoyama M. Protective effect of a mitochondria-targeted peptide against the development of chemotherapy-induced peripheral neuropathy in mice. ACS Chem Neurosci. 2018;9:1566-1571. doi:10.1021/ acschemneuro.8b00013

101. Zhao T, Liu X, Singh S, et al. Mitochondria penetrating peptideconjugated tamra for live-cell long-term tracking. Bioconjug Chem. 2019;30:2312-2316. doi:10.1021/acs.bioconjchem.9b00465

102. Huang Y, Li X, Sha H, et al. Tumor-penetrating peptide fused to a pro-apoptotic peptide facilitates effective gastric cancer therapy. Oncol Rep. 2017;37:2063-2070. doi:10.3892/or.2017.5440

103. Farsinejad S, Gheisary Z, Ebrahimi Samani S, Alizadeh AM. Mitochondrial targeted peptides for cancer therapy. Tumour Biol. 2015;36:5715-5725. doi:10.1007/s13277-015-3719-1

104. Panneerselvam P, Singh LP, Ho B, Chen J, Ding JL. Targeting of pro-apoptotic tlr adaptor sarm to mitochondria: definition of the critical region and residues in the signal sequence. Biochem $\mathrm{J}$. 2012;442:263-271. doi:10.1042/BJ20111653

105. Omura T. Mitochondria-targeting sequence, a multi-role sorting sequence recognized at all steps of protein import into mitochondria. J Biochem. 1998;123:1010-1016. doi:10.1093/oxfordjournals.jbchem.a022036

106. Sibrian-Vazquez M, Nesterova IV, Jensen TJ, Vicente MG. Mitochondria targeting by guanidine- and biguanidine-porphyrin photosensitizers. Bioconjug Chem. 2008;19:705-713. doi:10.1021/bc700393u

107. Lin F, Bao YW, Wu FG. Improving the phototherapeutic efficiencies of molecular and nanoscale materials by targeting mitochondria. Molecules. 2018;23(11):3016. doi:10.3390/molecules23113016

108. Ghayyem S, Faridbod F. A fluorescent aptamer/carbon dots based assay for cytochrome c protein detection as a biomarker of cell apoptosis. Methods Appl Fluoresc. 2018;7:015005. doi:10.1088/ 2050-6120/aaf0ca

109. Shamsipur M, Pashabadi A, Molaabasi F, Hosseinkhani S. Impedimetric monitoring of apoptosis using cytochrome-aptamer bioconjugated silver nanocluster. Biosens Bioelectron. 2017;90:195-202. doi:10.1016/j.bios.2016.11.050

110. Zhang W, Zhang T, Chen Y. Simultaneous quantification of cyt c interactions with hsp27 and bcl-xl using molecularly imprinted polymers (mips) coupled with liquid chromatography-tandem mass spectrometry (lc-ms/ms)-based targeted proteomics. $J$ Proteomics. 2019;192:188-195. doi:10.1016/j.jprot.2018.09.001

111. Zhu Q, Zhuang W, Chen Y, et al. Nano-biocatalysts of cyt c@zif8/go composites with high recyclability via a de novo approach. ACS Appl Mater Interfaces. 2018;10:16066-16076. doi:10.1021/ acsami.8b00072

112. Zhang PL, Wang ZK, Chen QY, Du X, Gao J. Biocompatible g-quadruplex/bodipy assembly for cancer cell imaging and the attenuation of mitochondria. Bioorg Med Chem Lett. 2019;29:1943-1947. doi:10.1016/j.bmcl.2019.05.043

113. Yamada Y, Furukawa R, Harashima H. A dual-ligand liposomal system composed of a cell-penetrating peptide and a mitochondrial RNA aptamer synergistically facilitates cellular uptake and mitochondrial targeting. J Pharm Sci. 2016;105:1705-1713. doi:10.1016/j.xphs.2016.03.002

114. Tawaraya Y, Hyodo M, Ara MN, Yamada Y, Harashima H. RNA aptamers for targeting mitochondria using a mitochondria-based selex method. Biol Pharm Bull. 2014;37:1411-1415. doi:10.1248/ bpb.b14-00112

115. Han C, Zhang C, Ma T, et al. Hypericin-functionalized graphene oxide for enhanced mitochondria-targeting and synergistic anticancer effect. Acta Biomater. 2018;77:268-281. doi:10.1016/j. actbio.2018.07.018
116. Hua X-W, Bao Y-W, Chen Z, Wu F-G. Carbon quantum dots with intrinsic mitochondrial targeting ability for mitochondria-based theranostics. Nanoscale. 2017;9:10948-10960. doi:10.1039/ C7NR03658B

117. Bae Y, Jung MK, Song SJ, et al. Functional nanosome for enhanced mitochondria-targeted gene delivery and expression. Mitochondrion. 2017;37:27-40. doi:10.1016/j.mito.2017.06.005

118. Weissig V. Dqasomes as the prototype of mitochondria-targeted pharmaceutical nanocarriers: preparation, characterization, and use. Methods Mol Biol. 2015;1265:1-11.

119. Gu Z, Zhu S, Yan L, Zhao F, Zhao Y. Graphene-based smart platforms for combined cancer therapy. Adv Mater. 2019;31: e1800662. doi:10.1002/adma.201800662

120. Liu JH, Li RS, Yuan B, Wang J, Li YF, Huang CZ. Mitochondriatargeting single-layered graphene quantum dots with dual recognition sites for ATP imaging in living cells. Nanoscale. 2018;10:17402-17408. doi:10.1039/C8NR06061D

121. Augustine R, Kalva N, Kim HA, Zhang Y, Kim I. Ph-responsive polypeptide-based smart nano-carriers for theranostic applications. Molecules. 2019;24:2961. doi:10.3390/ molecules24162961

122. Zhang ZT, Huang-Fu MY, Xu WH, Han M. Stimulus-responsive nanoscale delivery systems triggered by the enzymes in the tumor microenvironment. Eur J Pharm Biopharm. 2019;137:122-130. doi:10.1016/j.ejpb.2019.02.009

123. Fang Z, Li X, Xu Z, et al. Hyaluronic acid-modified mesoporous silica-coated superparamagnetic $\mathrm{fe}_{3} \mathrm{O}_{4}$ nanoparticles for targeted drug delivery. Int J Nanomedicine. 2019;14:5785-5797. doi:10.2147/IJN.S213974

124. Zhu X, Zhang B, Ye Z, Shi H, Shen Y, Li G. An ATP-responsive smart gate fabricated with a graphene oxide-aptamer-nanochannel architecture. Chem Commun (Camb). 2015;51:640-643. doi:10.1039/C4CC07990F

125. Cheng R, Feng F, Meng F, Deng C, Feijen J, Zhong Z. Glutathione-responsive nano-vehicles as a promising platform for targeted intracellular drug and gene delivery. J Control Release. 2011;152:2-12. doi:10.1016/j.jconrel.2011.01.030

126. Gao M, Liang C, Song X, et al. Erythrocyte-membrane-enveloped perfluorocarbon as nanoscale artificial red blood cells to relieve tumor hypoxia and enhance cancer radiotherapy. Adv Mater. 2017;29:1701429. doi:10.1002/adma.201701429

127. Cheng R, Meng F, Deng C, Klok HA, Zhong Z. Dual and multistimuli responsive polymeric nanoparticles for programmed sitespecific drug delivery. Biomaterials. 2013;34:3647-3657. doi:10.1016/j.biomaterials.2013.01.084

128. Fleige E, Quadir MA, Haag R. Stimuli-responsive polymeric nanocarriers for the controlled transport of active compounds: concepts and applications. Adv Drug Deliv Rev. 2012;64:866884. doi:10.1016/j.addr.2012.01.020

129. Swietach P, Vaughan-Jones RD, Harris AL, Hulikova A. The chemistry, physiology and pathology of ph in cancer. Philos Trans R Soc Lond B Biol Sci. 2014;369:20130099. doi:10.1098/ rstb.2013.0099

130. Cao L, Zhao Z, Zhang T, et al. In vivo observation of the ph alternation in mitochondria for various external stimuli. Chem Commun (Camb). 2015;51:17324-17327. doi:10.1039/C5CC07118F

131. Culcasi M, Casano G, Lucchesi C, et al. Synthesis and biological characterization of new aminophosphonates for mitochondrial ph determination by (31)p NMR spectroscopy. J Med Chem. 2013;56:2487-2499. doi:10.1021/jm301866e

132. Kato Y, Ozawa S, Miyamoto C, et al. Acidic extracellular microenvironment and cancer. Cancer Cell Int. 2013;13:89. doi:10.1186/1475-2867-13-89

133. Zhu YJ, Chen F. Ph-responsive drug-delivery systems. Chem Asian J. 2015;10:284-305. doi:10.1002/asia.201402715 
134. Pang X, Jiang Y, Xiao Q, Leung AW, Hua H, Xu C. Ph-responsive polymer-drug conjugates: design and progress. $J$ Control Release. 2016;222:116-129. doi:10.1016/j.jconrel.2015.12.024

135. Torchilin VP. Multifunctional, stimuli-sensitive nanoparticulate systems for drug delivery. Nat Rev Drug Discov. 2014;13:813827. doi: $10.1038 / \operatorname{nrd} 4333$

136. Yu F, Wu H, Tang Y, Xu Y, Qian X, Zhu W. Temperature-sensitive copolymer-coated fluorescent mesoporous silica nanoparticles as a reactive oxygen species activated drug delivery system. Int J Pharm. 2018;536:11-20. doi:10.1016/j.ijpharm.2017.11.025

137. Yue C, Yang Y, Zhang C, et al. Ros-responsive mitochondriatargeting blended nanoparticles: chemo- and photodynamic synergistic therapy for lung cancer with on-demand drug release upon irradiation with a single light source. Theranostics. 2016;6:23522366. doi:10.7150/thno. 15433

138. Reshetnikov V, Daum S, Janko C, et al. Ros-responsive n-alkylaminoferrocenes for cancer-cell-specific targeting of mitochondria. Angew Chem Int Ed Engl. 2018;57:11943-11946. doi:10.1002/anie.201805955

139. Liu D, Yang F, Xiong F, Gu N. The smart drug delivery system and its clinical potential. Theranostics. 2016;6:1306-1323. doi:10.7150/thno. 14858

140. Kim K, Choi ES, Choi ES, Park MH, Ryu JH. Hyaluronic acidcoated nanomedicine for targeted cancer therapy. Pharmaceutics. 2019;11:301. doi:10.3390/pharmaceutics11070301

141. Karimi M, Ghasemi A, Sahandi Zangabad P, et al. Smart micro/ nanoparticles in stimulus-responsive drug/gene delivery systems. Chem Soc Rev. 2016;45:1457-1501.

142. Maisonneuve L, Lamarzelle O, Rix E, Grau E, Cramail H. Isocyanate-free routes to polyurethanes and poly(hydroxy urethane)s. Chem Rev. 2015;115:12407-12439. doi:10.1021/acs. chemrev. 5 b00355

143. Mu J, Lin J, Huang P, Chen X. Development of endogenous enzyme-responsive nanomaterials for theranostics. Chem Soc Rev. 2018;47:5554-5573. doi:10.1039/C7CS00663B

144. Wang Y, Kohane DS. External triggering and triggered targeting strategies for drug delivery. Nat Rev Mater. 2017;2. doi:10.1038/ natrevmats.2017.20

145. Rahoui N, Jiang B, Taloub N, Huang YD. Spatio-temporal control strategy of drug delivery systems based nano structures. $J$ Control Release. 2017;255:176-201.

146. Kong L, Yang L, Xin CQ, et al. A novel flurophore-cyanocarboxylic-ag microhybrid: enhanced two photon absorption for two-photon photothermal therapy of hela cancer cells by targeting mitochondria. Biosens Bioelectron. 2018;108:14-19. doi:10.1016/ j.bios.2018.02.028

147. Sheng D, Liu T, Deng L, et al. Perfluorooctyl bromide \& indocyanine green co-loaded nanoliposomes for enhanced multimodal imaging-guided phototherapy. Biomaterials. 2018;165:1-13. doi:10.1016/j.biomaterials.2018.02.041

148. Luo S, Tan X, Fang S, et al. Mitochondria-targeted small-molecule fluorophores for dual modal cancer phototherapy. Adv Funct Mater. 2016;26:2826-2835. doi:10.1002/adfm.201600159

149. Raza A, Hayat U, Rasheed T, Bilal M, Iqbal HMN. "Smart" materials-based near-infrared light-responsive drug delivery systems for cancer treatment: a review. J Mater Res Technol. 2019;8:1497-1509. doi:10.1016/j.jmrt.2018.03.007

150. Freeman MW, Arrott A, Watson JHL. Magnetism in medicine. $J$ Appl Phys. 1960;31:S404-S405. doi:10.1063/1.1984765

151. Hossen S, Hossain MK, Basher MK, Mia MNH, Rahman MT, Uddin MJ. Smart nanocarrier-based drug delivery systems for cancer therapy and toxicity studies: a review. $J$ Adv Res. 2019;15:1-18.

152. Chen Z, Yin JJ, Zhou YT, et al. Dual enzyme-like activities of iron oxide nanoparticles and their implication for diminishing cytotoxicity. ACS Nano. 2012;6:4001-4012. doi:10.1021/nn300291r
153. Revia RA, Zhang M. Magnetite nanoparticles for cancer diagnosis, treatment, and treatment monitoring: recent advances. Mater Today (Kidlington). 2016;19:157-168. doi:10.1016/j.mattod.20 15.08.022

154. Jiang T, Zhang Z, Zhang Y, et al. Dual-functional liposomes based on ph-responsive cell-penetrating peptide and hyaluronic acid for tumor-targeted anticancer drug delivery. Biomaterials. 2012;33:9246-9258. doi:10.1016/j.biomaterials.2012.09.027

155. Zhang WY, Yi QY, Wang YJ, et al. Photoinduced anticancer activity studies of iridium(iii) complexes targeting mitochondria and tubules. Eur J Med Chem. 2018;151:568-584. doi:10.1016/j. ejmech.2018.04.013

156. He L, Wang KN, Zheng Y, et al. Cyclometalated iridium(iii) complexes induce mitochondria-derived paraptotic cell death and inhibit tumor growth in vivo. Dalton Trans. 2018;47:69426953. doi:10.1039/C8DT00783G

157. Huang H, Banerjee S, Qiu K, et al. Targeted photoredox catalysis in cancer cells. Nat Chem. 2019;11:1041-1048. doi:10.1038/ s41557-019-0328-4

158. Kawczyk-Krupka A, Bugaj AM, Latos W, Zaremba K, Wawrzyniec K, Sieron A. Photodynamic therapy in colorectal cancer treatment: the state of the art in clinical trials. Photodiagnosis Photodyn Ther. 2015;12:545-553. doi:10.1016/j. pdpdt.2015.04.004

159. Kohoutova D, Haidry R, Banks M, et al. Long-term outcomes of the randomized controlled trial comparing 5-aminolaevulinic acid and photofrin photodynamic therapy for barrett's oesophagus related neoplasia. Scand J Gastroenterol. 2018;53:527-532. doi:10.1080/00365521.2017.1403646

160. Lin YT, Hsiao YC, Chiang YF, Chang CJ. Topical application of photofrin((r)) for photodynamic diagnosis of malignant cutaneous neoplasms. J Plast Reconstr Aesthet Surg. 2018;71:1487-1495. doi:10.1016/j.bjps.2018.05.051

161. Lamch L, Bazylinska U, Kulbacka J, Pietkiewicz J, BiezunskaKusiak K, Wilk KA. Polymeric micelles for enhanced photofrin ii (r) delivery, cytotoxicity and pro-apoptotic activity in human breast and ovarian cancer cells. Photodiagnosis Photodyn Ther. 2014;11:570-585. doi:10.1016/j.pdpdt.2014.10.005

162. Ichikawa K, Takeuchi Y, Yonezawa S, et al. Antiangiogenic photodynamic therapy (pdt) using visudyne causes effective suppression of tumor growth. Cancer Lett. 2004;205:39-48. doi:10.1016/j.canlet.2003.10.001

163. Skupin-Mrugalska P, Piskorz J, Goslinski T, Mielcarek J, Konopka K, Duzgunes N. Current status of liposomal porphyrinoid photosensitizers. Drug Discov Today. 2013;18:776-784. doi:10.1016/j.drudis.2013.04.003

164. Rizvi I, Nath S, Obaid G, et al. A combination of visudyne and a lipid-anchored liposomal formulation of benzoporphyrin derivative enhances photodynamic therapy efficacy in a $3 \mathrm{~d}$ model for ovarian cancer. Photochem Photobiol. 2019;95:419-429. doi: $10.1111 /$ php. 13066

165. Inoue K. 5-aminolevulinic acid-mediated photodynamic therapy for bladder cancer. Int J Urol. 2017;24:97-101. doi:10.1111/ iju. 13291

166. Raharja PAR, Hamid A, Mochtar CA, Umbas R. Recent advances in optical imaging technologies for the detection of bladder cancer. Photodiagnosis Photodyn Ther. 2018;24:192-197. doi:10.1016/j.pdpdt.2018.10.009

167. McEwan C, Nesbitt H, Nicholas D, et al. Comparing the efficacy of photodynamic and sonodynamic therapy in non-melanoma and melanoma skin cancer. Bioorg Med Chem. 2016;24:3023-3028. doi:10.1016/j.bmc.2016.05.015

168. Mura S, Nicolas J, Couvreur P. Stimuli-responsive nanocarriers for drug delivery. Nat Mater. 2013;12:991-1003. doi:10.1038/ nmat3776 
169. Rao NV, Ko H, Lee J, Park JH. Recent progress and advances in stimuli-responsive polymers for cancer therapy. Front Bioeng Biotechnol. 2018;6:110. doi:10.3389/fbioe.2018.00110

170. Zinovkin RA, Zamyatnin AA. Mitochondria-targeted drugs. Curr Mol Pharmacol. 2019;12:202-214. doi:10.2174/187446721 2666181127151059

171. Rossman MJ, Santos-Parker JR, Steward CAC, et al. Chronic supplementation with a mitochondrial antioxidant (mitoq) improves vascular function in healthy older adults. Hypertension. 2018;71:1056-1063. doi:10.1161/HYPERTENSIONAHA.117.10 787

172. Pak O, Scheibe S, Esfandiary A, et al. Impact of the mitochondria-targeted antioxidant mitoq on hypoxia-induced pulmonary hypertension. Eur Respir J. 2018;51(3):1701024. doi:10.1183/ 13993003.01024-2017

173. Petrov A, Perekhvatova N, Skulachev M, Stein L, Ousler G. Skq1 ophthalmic solution for dry eye treatment: results of a phase 2 safety and efficacy clinical study in the environment and during challenge in the controlled adverse environment model. Adv Ther. 2016;33:96-115. doi:10.1007/s12325-015-0274-5

174. Cui Q, Wen S, Huang P. Targeting cancer cell mitochondria as a therapeutic approach: recent updates. Future Med Chem. 2017;9:929-949. doi:10.4155/fmc-2017-0011

175. Cardin DB, Thota R, Goff LW, et al. A Phase II study of ganetespib as second-line or third-line therapy for metastatic pancreatic cancer. Am J Clin Oncol. 2018;41:772-776. doi:10.1097/COC.0000000000000377

176. Subramaniam DS, Liu SV, Crawford J, et al. A phase ib/ii study of ganetespib with doxorubicin in advanced solid tumors including relapsed-refractory small cell lung cancer. Front Oncol. 2018;8:64. doi:10.3389/fonc.2018.00064
177. Meehan R, Kummar S, Do K, et al. A Phase I study of ganetespib and ziv-aflibercept in patients with advanced carcinomas and sarcomas. Oncologist. 2018;23:1269-e1125. doi:10.1634/theoncologist.2018-0203

178. Li J, Anraku Y, Kataoka K. Self-boosting catalytic nanoreactors integrated with triggerable crosslinking membrane networks for initiation of immunogenic cell death by pyroptosis. Angew Chem Int Ed Engl. 2020;59:13526-13530. doi:10.1002/anie.202004180

179. Li J, Li Y, Wang Y, et al. Polymer prodrug-based nanoreactors activated by tumor acidity for orchestrated oxidation/chemotherapy. Nano Lett. 2017;17:6983-6990. doi:10.1021/acs.nanolett. $7 \mathrm{~b} 03531$

180. Li J, Ke W, Wang L, et al. Self-sufficing h2o2-responsive nanocarriers through tumor-specific $\mathrm{h} 2 \mathrm{o} 2$ production for synergistic oxidation-chemotherapy. J Control Release. 2016;225:64-74. doi:10.1016/j.jconrel.2016.01.029

181. Singh AV, Maharjan RS, Kanase A, et al. Machine-learning-based approach to decode the influence of nanomaterial properties on their interaction with cells. ACS Appl Mater Interfaces. 2021;13:1943-1955. doi:10.1021/acsami.0c18470

182. Singh AV, Ansari MHD, Rosenkranz D, et al. Artificial intelligence and machine learning in computational nanotoxicology: unlocking and empowering nanomedicine. Adv Healthc Mater. 2020;9:e1901862. doi:10.1002/adhm.201901862

183. Singh AV, Rosenkranz D, Ansari MHD, et al. Artificial intelligence and machine learning empower advanced biomedical material design to toxicity prediction. Adv Intell Syst. 2020;2:2070125. doi:10.1002/aisy.202070125
International Journal of Nanomedicine

\section{Publish your work in this journal}

The International Journal of Nanomedicine is an international, peerreviewed journal focusing on the application of nanotechnology in diagnostics, therapeutics, and drug delivery systems throughout the biomedical field. This journal is indexed on PubMed Central, MedLine, CAS, SciSearch ${ }^{\mathbb{}}$, Current Contents ${ }^{\mathbb{R}} /$ Clinical Medicine, $^{2}$

\section{Dovepress}

Journal Citation Reports/Science Edition, EMBase, Scopus and the Elsevier Bibliographic databases. The manuscript management system is completely online and includes a very quick and fair peer-review system, which is all easy to use. Visit http://www.dovepress.com/ testimonials.php to read real quotes from published authors. 